\title{
Photoelectric Emission from Interstellar Dust: Grain Charging and Gas Heating
}

\author{
Joseph C. Weingartner \\ Physics Dept., Jadwin Hall, Princeton University, Princeton, NJ 08544, USA; CITA, 60 St. \\ George Street, University of Toronto, Toronto, ON M5S 3H8, Canada \\ weingart@cita.utoronto.ca \\ and \\ B.T. Draine \\ Princeton University Observatory, Peyton Hall, Princeton, NJ 08544, USA \\ draine@astro.princeton.edu
}

\begin{abstract}
We model the photoelectric emission from and charging of interstellar dust and obtain photoelectric gas heating efficiencies as a function of grain size and the relevant ambient conditions. Using realistic grain size distributions, we evaluate the net gas heating rate for various interstellar environments, and find less heating for dense regions characterized by $R_{V}=5.5$ than for diffuse regions with $R_{V}=3.1$. We provide fitting functions which reproduce our numerical results for photoelectric heating and recombination cooling for a wide range of interstellar conditions. In a separate paper we will examine the implications of these results for the thermal structure of the interstellar medium. Finally, we investigate the potential importance of photoelectric heating in $\mathrm{H}$ II regions, including the warm ionized medium. We find that photoelectric heating could be comparable to or exceed heating due to photoionization of $\mathrm{H}$ for high ratios of the radiation intensity to the gas density. We also find that photoelectric heating by dust can account for the observed variation of temperature with distance from the galactic midplane in the warm ionized medium.
\end{abstract}

Subject headings: dust — extinction — HII regions — ISM: clouds

\section{Introduction}

Photoelectric emission from dust grains dominates the heating of diffuse interstellar gas clouds as well as the photodissociation regions at the surfaces of molecular clouds. This mechanism therefore plays an important role in the dynamical evolution of the interstellar medium, and in 
shaping the line emission spectra of galaxies. Heating by photoelectric emission from interstellar grains was first considered by Spitzer (1948), and there have since been a number of reassessments of the heating rate (Watson 1972; deJong 1977; Draine 1978; Tielens \& Hollenbach 1985; Bakes \& Tielens 1994, Dwek \& Smith 1996).

For a given grain, the heating rate depends on the grain size, composition, and charge state, as well as on the spectrum of the illuminating radiation. Because photoelectric yields are enhanced for small grains (Watson 1972, 1973), estimates of the net photoelectric heating rate in interstellar gas are sensitive to the adopted grain size distribution, which should be consistent with the observed extinction curve (which shows strong regional variations) as well as with the observed emission from interstellar dust grains, from the near-infrared to the microwave. Previous estimates for photoelectric heating rates did not always use grain size distributions which were consistent with these constraints. While the measured extinction curve by itself does not suffice to uniquely specify the grain size distribution, in the present study we will consider size distributions which are consistent with the observed extinction in different regions, with either the minimum or maximum permissible population of ultrasmall grains (Weingartner \& Draine 2000).

In addition to using size distributions consistent with observations, we also model the photoelectric emission process and associated grain charging in detail, using realistic yields for graphitic and silicate grains, a realistic distribution of photoelectron kinetic energies, and new estimates for electron sticking efficiencies for small grains. The resulting photoelectric heating rates are calculated for grain size distributions consistent with extinction curves characteristic of diffuse clouds $\left(R_{V} \equiv A_{V} / E_{B-V} \approx 3.1\right)$, intermediate density regions $\left(R_{V} \approx 4.0\right)$, and dense clouds $\left(R_{V} \approx 5.5\right)$.

In $\S 2$ we discuss our adopted model for photoelectric emission from grains, expanding on the treatments of Draine (1978) and Bakes \& Tielens (1994). In $\S 3$ we use recent experimental results to obtain improved estimates for the electron sticking coefficient, as a function of grain size; our new sticking coefficients differ significantly from previous estimates. In $\S 4$ we characterize the relevant ambient conditions (which are important in grain charging) and in $\S 5$ we evaluate the photoelectric heating efficiency as a function of grain size. We obtain net heating rates in $\mathrm{H}$ I regions in $\S 6$ and in $\S 7$ we investigate the potential contribution of photoelectric heating in H II regions, finding that grain photoelectric heating may explain observed high temperatures in the warm ionized medium. We briefly summarize our results in $\S 8$.

\section{Photoelectric Emission from Grains}

\subsection{Grain Characterization}

We consider both graphitic and silicate grains. We adopt the description of graphitic grains given by Li \& Draine (2000), in which the smallest grains are polycyclic aromatic hydrocarbon (PAH) molecules, the largest grains consist of graphite, and grains of intermediate size have optical 
properties intermediate between those of PAHs and graphite. We characterize the graphitic grains by an effective radius $a$, the radius of a sphere containing the same number of $\mathrm{C}$ atoms, in which $\mathrm{C}$

atoms contribute a mass density $\rho=2.24 \mathrm{~g} \mathrm{~cm}^{-3}$, the density of ideal graphite. The smallest grains certainly are not spherical and probably are not well characterized by the ideal graphite density; in this case the effective radius simply indicates the number $N_{\mathrm{C}}$ of $\mathrm{C}$ atoms in the grain:

$$
N_{\mathrm{C}}=468\left(\frac{a}{10^{-7} \mathrm{~cm}}\right)^{3}
$$

For example, coronene $\mathrm{C}_{24} \mathrm{H}_{12}$ is assigned an effective radius $a=3.7 \AA$, and a surface area $4 \pi a^{2}=$ $173 \AA^{2}$.

In following sections, the $N_{\mathrm{C}}-a$ relation (1) will be used to incorporate experimental data on hydrocarbon molecules into our description in terms of the effective radius. Since small molecules that extrapolate to silicates as $a \rightarrow \infty$ have not been studied, we will not need a similar relation for silicates. In this case, $a$ is simply the radius of a spherical silicate grain.

\subsection{Electron Energy Levels}

The electrons in a solid are confined in a potential well (see, e.g., Lang \& Kohn 1971). For neutral bulk material, the work function $W$ is the energy of a free electron with zero kinetic energy minus the energy of an electron in the highest occupied state in the solid. (Throughout this section, refer to Figure 1 for schematic depictions of the electron-confining potential.) In this case, the ionization potential $I P$, i.e., the difference in energy between infinity and the highest occupied state, equals $W$.

For small grains, $I P \neq W$. We first consider grains which are not so small that quantum effects shift the energy levels from their bulk values. If $Z \geq 0$, then an electron that is liberated from the grain material feels a Coulomb attraction to the grain, which now has charge $(Z+1) e$. For a conducting sphere, $I P-W$ can be determined by calculating the work needed to remove the electron from a point just outside the sphere to infinity (including the term due to the image charge as well as that due to the net charge on the sphere) and subtracting the corresponding work associated with a plane surface. The result is that $I P=W+(Z+1 / 2) e^{2} / a$ (see, e.g. Makov, Nitzan, \& Brus 1988; Seidl \& Brack 1996). ${ }^{1}$ For nonconducting materials, the term $e^{2} / 2 a$ above should be multiplied by $(\epsilon-1) / \epsilon$, where $\epsilon$ is the dielectric function (Makov et al. 1988). This correction is fairly small for candidate grain materials, so we will ignore it.

When $Z<0$, the "extra" electrons, which occupy the "LUMO" (for "lowest unoccupied molecular orbital") of the neutral, are separated from the top of the valence band by the band

\footnotetext{
${ }^{1}$ Surprisingly, there has been some controversy in the literature as to the value of the constant added to $Z$. See Makov et al. (1988), Moskovits (1991), Seidl \& Brack (1996).
} 
gap, with extra energy $E_{\mathrm{bg}}$ (assuming the valence band is full in the neutral). The electron affinity $E A(Z)$ is the difference in energy between infinity and the LUMO for the grain of charge $(Z-1) e$ created by adding the electron. Thus, $\operatorname{IP}(Z<0)=E A(Z+1)$. We also define the "valence band ionization potential" $I P_{V}(Z)$, equal to the difference in energy between infinity and the top of the valence band. Of course, $I P_{V}(Z)=I P(Z)$ when $Z \geq 0$.

Since it is not yet possible to calculate the quantum shifts in energy levels for very small grains, we adopt an empirical approach. Figure 2 shows the first and second ionization potentials $I P(0)$ and $I P(1)$ for various aromatic hydrocarbons, including $\mathrm{C}_{6} \mathrm{H}_{6}$ benzene, $\mathrm{C}_{24} \mathrm{H}_{12}$ coronene, and $\mathrm{C}_{38} \mathrm{H}_{20}$ benz[42]. The polycyclic aromatic hydrocarbons (PAHs) of interest here appear to be fit by

$$
I P_{V}(Z)=W+\left(Z+\frac{1}{2}\right) \frac{e^{2}}{a}+(Z+2) \frac{e^{2}}{a}\left(\frac{0.3 \AA}{a}\right),
$$

with the graphite work function $W=4.4 \mathrm{eV}$. This estimate for $I P(Z)$ is plotted in Figure 2 for $Z=0$ and 1 . For the first ionization potential $I P(0)$, equation $(2)$ is numerically very close to the estimates of Bakes (1992) and Bakes and Tielens (1994), but for IP(1) our estimate is $\sim 1 \mathrm{eV}$ below their estimate for PAHs with $\sim 15-30 \mathrm{C}$ atoms. Note that equation (2) reproduces the classical electrostatic result for large $a$. Since experimental evidence is not available for other ionization states, we will adopt equation (2) for all values of $Z$.

For silicates, few laboratory experiments are available to guide us. We adopt $W=8 \mathrm{eV}$, since the photoelectric yield for lunar dust drops steeply around that energy (Feuerbacher et al. 1972). Nayak et al. (1998) have made theoretical calculations of the ionization potentials of small silica clusters $\left(\mathrm{SiO}_{2}\right)_{n}$, with $n=1$ to 6 . They find $I P(0)=11.19 \mathrm{eV}(16.08 \mathrm{eV})$ for $n=2(5)$, and intermediate results for other values of $n$. If we employ equation (2) with $W=8 \mathrm{eV}$ and $a \approx 3.2 \AA$, then we find $I P(0) \approx 12 \mathrm{eV}$, which is in the Nayak et al. range for small clusters. Thus, we adopt equation (2) for both carbonaceous and silicate grains, with $W=4.4$ and $8 \mathrm{eV}$, respectively.

We expect that

$$
E A(Z \leq 0)=W-E_{\mathrm{bg}}(a)+\left(Z-\frac{1}{2}\right) \frac{e^{2}}{a}+O\left(a^{-2}\right)
$$

where the presence of an $O\left(a^{-2}\right)$ term is expected since such a correction was required to fit the experimental results for $I P_{V}(Z \geq 0)$ (eq. 2). The "infinite limit" for PAHs - graphite - is a "semimetal", with a slight overlap between the top of the valence band and the conduction band (Spain 1973). For metals and semimetals, $E_{\mathrm{bg}}=0$. Many silicates begin to absorb strongly around 2500-2000 $\AA$, corresponding to a band gap of $5-6 \mathrm{eV}$; we will adopt $E_{\mathrm{bg}}=5 \mathrm{eV}$, independent of $a$, for silicates.

Values of $E A(0)$ are shown in Figure 3 for selected neutral PAHs, benzene, and $\mathrm{C}_{60}$. The relation

$$
E A(Z)=W+\left(Z-\frac{1}{2}\right) \frac{e^{2}}{a}-\frac{e^{2}}{a}\left(\frac{4 \AA}{a+7 \AA}\right)
$$


is also plotted in Figure 3, for $Z=0$ and $W=4.4 \mathrm{eV}$. For $a \rightarrow \infty$ this has the theoretically expected behavior, and it provides a reasonable fit to the experimental values of $E A(0)$ for benzene and the PAHs in Figure 3 (for which $a \approx 2.3-4.3 \AA$ ). We adopt this equation (4) for carbonaceous grains. For silicates, we adopt

$$
E A(Z)=W-E_{\mathrm{bg}}+\left(Z-\frac{1}{2}\right) \frac{e^{2}}{a}
$$

with $W-E_{\mathrm{bg}}=3 \mathrm{eV}$. This yields $E A(0) \approx 0.73 \mathrm{eV}$ for $a \approx 3.2 \AA$, which is somewhat low compared with the values of $2-3 \mathrm{eV}$ inferred for small silica clusters (Wang et al. 1997).

\subsection{Photoelectric Yields}

The photoelectric yield is the probability that an electron will be ejected following the absorption of a photon. Calculation of the yield from first principles is not yet possible. Draine (1978) displayed yield measurements from experiments on bulk samples of materials of interest, including graphite, lunar dust, and silicon carbide. Watson $(1972,1973)$ pointed out that the yields of submicron particles are expected to be enhanced relative to the bulk yields, because of the finite electron escape length. An electron excited somewhere in the volume of the sample can lose energy during its journey to the surface, through interactions with electrons and with phonons. The result is that the fraction of electrons which escape energy loss goes roughly as $\exp \left(-x / l_{e}\right)$, where $x$ is the distance the electron has traveled and $l_{e}$ is the "electron escape length." Generally, the photon attenuation length, $l_{a}$ (equal to the $e$-folding length for the decrease in radiation intensity as it propagates into the material), exceeds $l_{e}$, and the electrons excited deep inside a bulk sample do not reach the surface. Small grain sizes limit the distance from electron excitation to surface, so the yield is enhanced.

A detailed and accurate model for the above geometrical effect is not yet available. A series of experiments (Schmidt-Ott et al. 1980; Burtscher \& Schmidt-Ott 1982; Burtscher et al. 1984; Müller et al. 1988) on free silver spheres with radii between 27 and $54 \AA$ found yield enhancements in excess of expectations by factors of several. A later theoretical effort (Faraci, Pennisi, \& Margaritondo 1989) was able to reproduce the results, but only by assuming that the condition for an electron to escape, when incident on the surface from within, depends only on its energy and not on its direction of motion. The estimates adopted here for size-dependent yields from grains are based on Watson's (1973) simplified model for the geometrical effect and must be regarded as provisional. Reliable calculations of effects involving photoelectric emission from interstellar dust will not be possible until experiments have been performed on submicron grains of appropriate composition.

For negatively charged grains we will distinguish between "photoelectric" ejection of electrons from the valence band and "photodetachment" of excess "attached" electrons. 


\subsubsection{Valence Band Electrons}

We assume that, when $Z>0$, the highest occupied energy level is very close to the top of the valence band, since the number of electrons which have been removed from the grain is small compared with the total number of electrons in the grain. Thus, for $Z \geq 0$, the threshold photon energy for photoelectric emission is given by $h \nu_{\text {pet }}(Z)=I P_{V}(Z)=I P(Z)$. When $Z<-1$, $h \nu_{\text {pet }}(Z)>I P(Z)$, because the electron has to overcome the repulsive Coulomb barrier. Suppose the tunneling probability becomes significant when the electron energy exceeds the potential at infinity by $E_{\min }$. Then $h \nu_{\text {pet }}(Z)=I P_{V}(Z)+E_{\min }$. Thus, we take

$$
h \nu_{\text {pet }}(Z, a)=\left\{\begin{array}{ll}
I P_{V}(Z, a) & , Z \geq-1 \\
I P_{V}(Z, a)+E_{\min }(Z, a) & , Z<-1
\end{array} .\right.
$$

We estimate $E_{\min }(Z, a)$ as the energy for which the tunneling probability (evaluated using the WKB approximation) equals $10^{-3}$; the tunneling probability rapidly increases as the excited electron energy increases above this value. The barrier consists of the Coulomb potential $-(Z+$ 1) $e^{2} / r$ and the image potential $-e^{2} a^{3} / 2 r^{2}\left(r^{2}-a^{2}\right)$, where $r$ is the distance from the center of the spherical grain. ${ }^{2}$ We adopt the following expression for $E_{\min }$, which reproduces the results of the WKB calculation fairly well when $-(Z+1) e^{2} / a \gtrsim 0.5 \mathrm{eV}$ :

$$
E_{\min }(Z<0, a)=-(Z+1) \frac{e^{2}}{a}\left[1+\left(\frac{27 \AA}{a}\right)^{0.75}\right]^{-1} .
$$

When $-(Z+1) e^{2} / a<1 \mathrm{eV}$, equation (7) overestimates $E_{\min }$ as defined above, with increasing severity as $-(Z+1) e^{2} / a \rightarrow 0$. However, in this limit $E_{\min } \rightarrow 0$ as well, so that this overestimate will not substantially affect the photoemission calculations.

We adopt a very simple model for estimating the photoelectric yield $Y(h \nu, Z, a)$. We first consider the yield $y_{0}^{\prime}$ of electrons that traverse the surface layer, emerge from the grain surface, and have enough energy to overcome the image potential (we will call these "attempting" electrons). For $Z<0$, every attempting electron will escape, but when $Z \geq 0$, some of these electrons have insufficient energy to escape to infinity and instead fall back to the grain. We assume the following form for $y_{0}^{\prime}$ :

$$
y_{0}^{\prime}=y_{0}(\Theta) y_{1}(h \nu, a)
$$

where the parameter $\Theta$ is given by

$$
\Theta= \begin{cases}h \nu-h \nu_{\text {pet }}+(Z+1) e^{2} / a & , Z \geq 0 \\ h \nu-h \nu_{\text {pet }} & , Z<0\end{cases}
$$

\footnotetext{
${ }^{2}$ Within a few $\AA$ of the surface, the image potential deviates from the classical expression, and "saturates" to a constant value (see, e.g., Lang \& Kohn 1971). However, this saturated portion of the potential is always classically allowed, and therefore does not affect the WKB estimate of the tunneling probability.
} 
and the factor $y_{1}(h \nu, a)$ accounts for the size-dependent geometrical yield enhancement discussed above; $y_{1}$ depends on $h \nu$ because the photon attenuation length $l_{a}$ does.

We assume a parabolic energy distribution for the attempting electrons: ${ }^{3}$

$$
f_{E}^{0}(E)=\frac{6\left(E-E_{\text {low }}\right)\left(E_{\text {high }}-E\right)}{\left(E_{\text {high }}-E_{\text {low }}\right)^{3}},
$$

where $f_{E}^{0}(E) d E$ gives the fraction of attempting electrons with energy (with respect to infinity) between $E$ and $E+d E$. When $Z<0, E_{\text {low }}=E_{\text {min }}$ (eq. 7) and $E_{\text {high }}=E_{\text {min }}+h \nu-h \nu_{\text {pet }}$; when $Z \geq 0, E_{\text {low }}=-(Z+1) e^{2} / a$ and $E_{\text {high }}=h \nu-h \nu_{\text {pet }}$.

Let $y_{2}$ be the fraction of attempting electrons which escape to infinity:

$$
y_{2}(h \nu, Z, a)= \begin{cases}\int_{0}^{E_{\text {high }}} d E f_{E}^{0}(E)=E_{\text {high }}^{2}\left(E_{\text {high }}-3 E_{\text {low }}\right) /\left(E_{\text {high }}-E_{\text {low }}\right)^{3} & , Z \geq 0 \\ 1 & , Z<0\end{cases}
$$

Our resulting expression for the photoelectric yield is

$$
Y(h \nu, Z, a)=y_{2}(h \nu, Z, a) \min \left[y_{0}(\Theta) y_{1}(a, h \nu), 1\right] \quad .
$$

Draine (1978) found that the following estimate for $y_{1}$ reproduces Watson's (1973) detailed results based on Mie theory, to within $20 \%$ :

$$
y_{1}=\left(\frac{\beta}{\alpha}\right)^{2} \frac{\alpha^{2}-2 \alpha+2-2 \exp (-\alpha)}{\beta^{2}-2 \beta+2-2 \exp (-\beta)},
$$

where $\beta=a / l_{a}$ and $\alpha=a / l_{a}+a / l_{e}$.

Electron escape lengths for most materials reach a minimum in the vicinity of tens of $\mathrm{eV}$ and increase at higher and lower energies. The data are meager for electron energies below $10 \mathrm{eV}$, but Martin et al. (1987) found, for thin carbon films, that $l_{e}$ fell into a broad minimum of $6 \AA$ around $40 \mathrm{eV}$ and rose to $9 \AA$ at $6 \mathrm{eV}$. McFeely et al. (1990) found, for $\mathrm{SiO}_{2}$, that $l_{e}$ varied from 5.7 to $6.8 \AA$ for electron energies between 8 and $20 \mathrm{eV}$, and one might expect similar values for silicates. Bakes \& Tielens (1994) assumed $l_{e}=10 \AA$, independent of energy, and we use the same value here, for both graphite and silicates.

The photon attenuation length is given by

$$
l_{a}=\frac{\lambda}{4 \pi \operatorname{Im}(m)}
$$

where $\lambda$ is the wavelength in vacuo and $m(\lambda)$ is the complex refractive index. Graphite is a highly anisotropic material, so that the dielectric function is a tensor. This tensor may be diagonalized

\footnotetext{
${ }^{3}$ A parabolic form is consistent with typical laboratory photoelectron distributions which drop to zero when $E=0$ and $E=h \nu-W$ and peak somewhere in between (Draine 1978).
} 
by choosing Cartesian coordinates with two of the axes lying in the "basal" plane; the third axis, normal to the basal plane, is called the " $c$-axis". For graphite, we take

$$
l_{a}^{-1}=\frac{4 \pi}{\lambda}\left[\frac{2}{3} \operatorname{Im}\left(m_{\perp}\right)+\frac{1}{3} \operatorname{Im}\left(m_{\|}\right)\right],
$$

where $m_{\perp}$ and $m_{\|}$are for the electric field perpendicular and parallel to the $c$-axis, respectively. In computing $l_{a}$, we use dielectric functions from Draine \& Lee (1984) and Laor \& Draine (1993), modified to remove a silicate feature of crystalline origin that is not present in the observed interstellar extinction or polarization (see Weingartner \& Draine 2000). Since PAH dielectric functions are not available, we use graphite dielectric functions for all carbonaceous grains.

The final step in our yield prescription is to estimate $y_{0}(\Theta)$ for carbonaceous and silicate grains. For carbonaceous grains, we follow Bakes \& Tielens (1994) and choose $y_{0}$ such that $Y(h \nu, Z=0, a=$ $3.7 \AA$ ) approximately reproduces the photoionization yield for coronene, as measured by Verstraete et al. (1990). This gives

$$
y_{0}(\Theta)=\frac{9 \times 10^{-3}(\Theta / W)^{5}}{1+3.7 \times 10^{-2}(\Theta / W)^{5}},
$$

with $W=4.4 \mathrm{eV}$ (see Figure 4). Draine (1978) shows, in his Figure 1, that the bulk graphite yield measured by Feuerbacher \& Fitton (1972) is unusually low, and lies more than an order of magnitude below the yield for anthracene (Fujihira, Hirooka, \& Inokuchi 1973). It is important to note that our equation (16) gives yields that substantially exceed those measured for bulk graphite. ${ }^{4}$ This apparent inconsistency could imply one or more of the following: 1. The yield for bulk graphite differs substantially from that for bulk coronene. 2. The measured yields for bulk graphite are wrong. 3. Our prescription for $y_{1}(a, h \nu)$ is wrong. Clearly, the adopted yields for carbonaceous grains are highly uncertain.

The situation for silicates is no better. Feuerbacher et al. (1972) measured the yield for a powdered sample of lunar dust, and found that $Y$ decreases rapidly as photon energy is decreased below $14 \mathrm{eV}$. However, they noted that the use of the powdered sample might result in yields that are too low. Thus, we choose $y_{0}$ for silicates such that the bulk yield somewhat exceeds the results of Feuerbacher et al. at $14 \mathrm{eV}$, but does not drop as rapidly for lower values of $h \nu$ :

$$
y_{0}(\Theta)=\frac{0.5(\Theta / W)}{1+5(\Theta / W)}
$$

with $W=8 \mathrm{eV}$.

In Figure 5 we plot $Y(h \nu)$ for neutral carbonaceous and silicate grains of several sizes.

\footnotetext{
${ }^{4}$ For example, at $h \nu=10 \mathrm{eV}$, where Feuerbacher \& Fitton (1972) measured a yield of $8 \times 10^{-3}$ for bulk graphite, our equation (16) gives $y_{0}=2.7 \times 10^{-2}$, a factor 3.4 larger than the laboratory value. For comparison, the Bakes \& Tielens (1994) expression gives $y_{0}=7.8 \times 10^{-2}$, an order of magnitude larger than the experimental value.
} 


\subsubsection{Photoelectric Ejection of Attached Electrons (Photodetachment)}

When $Z<0$, the $-Z$ attached electrons occupy energy levels above the valence band, if the latter is full in the neutral. We take the photodetachment threshold energy $h \nu_{\text {pdt }}$ to be

$$
h \nu_{\mathrm{pdt}}(Z<0)=E A(Z+1, a)+E_{\min }(Z, a)
$$

with electron affinities $E A$ given by equations (4) and (5), and $E_{\min }$ by (7). Bakes \& Tielens (1994) neglected $E_{\min }(Z, a)$ and took $h \nu_{\mathrm{pdt}}=E A(Z+1, a)$. We assume that $E=h \nu-h \nu_{\mathrm{pdt}}$ for photodetached electrons, since attached electrons lie in a narrow range of energies.

We assume that an oscillator strength $f_{\text {pdt }}$ is associated with photodetachment transitions to the continuum. The photodetachment cross section $\sigma_{\text {pdt }}(h \nu)$ has been measured for $\mathrm{C}_{6} \mathrm{~F}_{6}^{-}$ (Christophorou, Datskos, \& Faidas 1994). The measured cross section has considerable structure, but can be roughly approximated by

$$
\sigma_{\mathrm{pdt}}(Z, a)=-Z \frac{2 \pi e^{2} h f_{\mathrm{pdt}}}{3 m_{e} c \Delta E} \frac{x}{\left(1+x^{2} / 3\right)^{2}}
$$

where $x \equiv\left(h \nu-h \nu_{\mathrm{pdt}}\right) / \Delta E$ and the peak in $\sigma_{\text {pdt }}$ occurs at $h \nu=h \nu_{\mathrm{pdt}}+\Delta E$. We take $\Delta E=3 \mathrm{eV}$ and oscillator strength $f_{\mathrm{pdt}}=0.5$; thus

$$
\sigma_{\mathrm{pdt}}(h \nu, Z, a)=1.2 \times 10^{-17} \mathrm{~cm}^{2}|Z| \frac{x}{\left(1+x^{2} / 3\right)^{2}} \quad \text { for } Z<0 .
$$

\subsection{Grain Charging}

Since the photoemission depends on the grain charge, it is necessary to know the distribution of charge states for the grains. In statistical equilibrium,

$$
f_{Z}(Z)\left[J_{\mathrm{pe}}(Z)+J_{\mathrm{ion}}(Z)\right]=f_{Z}(Z+1) J_{e}(Z+1),
$$

where $f_{Z}(Z)$ is the probability for the grain charge to be $Z e, J_{\text {pe }}$ is the photoemission rate, $J_{\text {ion }}$ is the positive ion accretion rate, and $J_{e}$ is the electron accretion rate.

Bakes \& Tielens (1994) discuss the most positive and most negative charges that a grain could possibly acquire $\left(Z_{\max } e\right.$ and $Z_{\min } e$, respectively). The most positive charge is one proton charge

more than the highest charge for which an electron can be ejected, i.e. for which $h \nu_{\text {pet }}=I P<$ $h \nu_{\max }$, the maximum photon energy in the radiation field $(=13.6 \mathrm{eV}$, in an $\mathrm{H}$ I region). Thus,

$$
Z_{\max }=\operatorname{int}\left[\left(\frac{h \nu_{\max }-W}{14.4 \mathrm{eV}} \frac{a}{\AA}+\frac{1}{2}-\frac{0.3 \AA}{a}\right)\left(1+\frac{0.3 \AA}{a}\right)^{-1}\right],
$$

where int $[x]$ denotes the greatest integer less than $x$. The minimum allowed charge $Z_{\min } e$ is the most negative charge for which autoionization does not occur. Bakes \& Tielens (1994) assume 
that this requires $E A\left(Z_{E A}+1\right)>0$. However, large grains can be charged more negatively without emitting a substantial electron current when the tunneling probability is low. We take the autoionization threshold potential $U_{\text {ait }}$ to be that at which the electron current is $\approx 10^{-6} \mathrm{~s}^{-1}$; we evaluate the tunneling probability using the WKB approximation and assume an attempt frequency of $\approx 2 \times 10^{8} \mathrm{~s}^{-1}(\mathrm{~cm} / a)$. We find

$$
\frac{-U_{\text {ait }}}{\mathrm{V}} \approx\left\{\begin{array}{ll}
3.9+0.12(a / \AA)+2(\AA / a) & , \text { for carbonaceous } \\
2.5+0.07(a / \AA)+8(\AA / a) & , \text { for silicate }
\end{array} ;\right.
$$

this agrees very well with Draine \& Salpeter's (1979) simple estimate of the potential at which field emission becomes important. The most negative allowed charge is then given by

$$
Z_{\text {min }}=\operatorname{int}\left[\frac{U_{\text {ait }}}{14.4 \mathrm{~V}} \frac{a}{\AA}\right]+1
$$

For carbonaceous grains, equations (23)-(24) give $Z_{\min }=0$ for $a<2.92 \AA, Z_{\min }=-1$ for $2.92 \leq$ $a<5.75 \AA, Z_{\min }=-2$ for $5.75 \leq a<8.40 \AA$. For silicate grains, $Z_{\min }=0$ for $a<2.40 \AA$, $Z_{\min }=-1$ for $2.40 \leq a<6.96 \AA, Z_{\min }=-2$ for $6.96 \leq a<10.8 \AA$. By iteratively applying equation (21) and normalizing, $f_{Z}$ can be found for all $Z$.

The photoemission rate is given by

$$
J_{\mathrm{pe}}=\pi a^{2} \int_{\nu_{\mathrm{pet}}}^{\nu_{\max }} d \nu Y Q_{\mathrm{abs}}(\nu) \frac{c u_{\nu}}{h \nu}+\int_{\nu_{\mathrm{pdt}}}^{\nu_{\max }} d \nu \sigma_{\mathrm{pdt}}(\nu) \frac{c u_{\nu}}{h \nu},
$$

where $Q_{\mathrm{abs}}$ is the absorption efficiency, $u_{\nu}$ is the radiation energy density per frequency interval, and $c$ is the speed of light. The second term in equation (25) is only present when $Z<0$. Bakes \& Tielens (1994) assumed $Q_{\mathrm{abs}} \propto a$, valid for grains with $a \lesssim 100 \AA$. Since we consider larger grains, we cannot adopt this approximation. We evaluate $Q_{\text {abs }}$ for graphitic grains using the prescription of Li \& Draine $(2000)^{5}$ and for silicate grains using a Mie theory code derived from BHMIE (Bohren \& Huffman 1983) with dielectric functions given by Draine \& Lee (1984) and Laor \& Draine (1993), but modified in the ultraviolet (Weingartner \& Draine 2000).

The accretion rates are given by

$$
J_{i}(Z)=n_{i} s_{i}(Z)\left(\frac{8 k T}{\pi m_{i}}\right)^{1 / 2} \pi a^{2} \tilde{J}\left(\tau_{i}, \xi_{i}\right),
$$

where $n_{i}$ is the number density of species $i$, the sticking coefficient $s_{i}(Z)$ is the probability that species $i$ will transfer its charge if it reaches the surface of a grain of charge $Z e, m_{i}$ is the particle mass, $T$ is the gas temperature, and $\tilde{J}$ is a function of $\tau_{i} \equiv a k T / q_{i}{ }^{2}$ and $\xi_{i} \equiv Z e / q_{i}\left(q_{i}\right.$ is the charge of species $i$ and $k$ is the Boltzmann constant). Expressions for $\tilde{J}$ can be found in Draine \& Sutin (1987). The electron and ion sticking coefficients $s_{e}$ and $s_{i}$ are discussed below in $\S 3$.

\footnotetext{
${ }^{5} \mathrm{Li} \&$ Draine (2000) give optical properties for neutral and ionized grains; we use the former when $Z=0$ and the latter when $Z \neq 0$. The distinction is not critical, as in the vacuum ultraviolet there is little difference between the ionized and neutral grain absorption cross sections.
} 


\section{Sticking Coefficients}

\subsection{Electrons}

A low-energy electron impinging on a macroscopic solid surface has some probability $p_{\text {es }}$ of elastic scattering. We will assume that $p_{e s} \approx 0.5$, so that the maximum possible value of the sticking coefficient $s_{e}$ would be $\left(1-p_{e s}\right) \approx 0.5$.

\subsubsection{Attachment to Neutral Grains}

We first consider electron attachment to neutral grains. If the electron affinity $E A>0$ [as it is for $a>3.5 \AA$, which Weingartner \& Draine (2000) take to be the lower cutoff for the grain size distribution], then the approaching electron accelerates due to its polarization of the grain, arriving at the surface with a kinetic energy of order $E A$. Even if it enters the grain material [with probability $\left(1-p_{e s}\right)$ ], the electron may fail to undergo an inelastic scattering event, in which case it passes through the grain and returns to infinity. The probability of undergoing inelastic scattering is approximately $\left(1-e^{-a / l_{e}}\right)$, where $l_{e} \approx 10 \AA$, the "electron escape length" (discussed above), is roughly the mean free path against inelastic scattering within the grain material.

If the impinging electron does undergo inelastic scattering, then it transfers some of its energy to internal degrees of freedom of the grain; if the energy so transferred exceeds the initial kinetic energy $(\sim 2 k T)$, then the electron is trapped by the grain potential. However, until the grain radiates this energy away, there is a nonzero probability per unit time that the internal degrees of freedom of the grain will transfer enough energy back to the electron to eject it. Let $p_{\text {rad }}$ be the probability of "radiative stabilization" of the negatively charged grain, i.e. the probability of radiating away $\sim k T$ of energy before the electron is ejected. We expect $p_{\text {rad }} \rightarrow 1$ for macroscopic grains, but $p_{\text {rad }}<1$ for grains with a small number of internal degrees of freedom. The electron attachment sticking coefficient can then be written as the product of three factors:

$$
s_{e}(Z=0) \approx\left(1-p_{e s}\right)\left(1-e^{-a / l_{e}}\right) p_{\text {rad }} .
$$

The stabilization probability $p_{\text {rad }}$ can be estimated from the electron affinity $E A$, the density of states of the excited negatively charged grain created by the electron attachment, and the probability per time of photon emission from the excited grain (Allamandola, Tielens, \& Barker 1989; Tielens 1993). However, since these parameters are poorly known, we will instead adopt an empirical approach.

In Figure 6 we show sticking coefficients $s_{e}=\langle\sigma v\rangle /\left[\left(8 k T / \pi m_{e}\right)^{1 / 2} \pi a^{2} \tilde{J}\right]$ for various hydrocarbon molecules, where $\tilde{J}\left(a k T / e^{2}, 0\right)$ is given by Draine \& Sutin (1987) and the rate coefficients $\langle\sigma v\rangle$ for electron attachment are determined experimentally. Also shown in Figure 6 is a semiempirical fit:

$$
s_{e}(Z=0)=0.5\left(1-e^{-a / l_{e}}\right) \frac{1}{1+e^{\left(20-N_{\mathrm{C}}\right)}},
$$


where $N_{\mathrm{C}}$ is the number of atoms other than $\mathrm{H}$ in the molecule, and $l_{e}=10^{-7} \mathrm{~cm}$.

The measured sticking coefficients for $N_{\mathrm{C}} \leq 20$ are in reasonable agreement with our semiempirical fit (eq. 28). It is notable that Tobita et al. (1992) found that in many cases the sticking coefficient increases when the electron energy increases. For example, the sticking coefficients for tetracene $\mathrm{C}_{18} \mathrm{H}_{12}$ and perylene $\mathrm{C}_{20} \mathrm{H}_{12}$ would be larger by an order of magnitude for gas temperature $T \approx 2000 \mathrm{~K}$, bringing them up to or even above the values given by equation (28).

The measured $s_{e}$ for $\mathrm{C}_{60}$ and $\mathrm{C}_{70}$ at $T \leq 500 \mathrm{~K}$ are remarkably low, probably due to the unusual rigidity and symmetry of these molecules. For $\mathrm{C}_{60}$, the symmetry forbids capture of $s$-wave electrons, and the centrifugal barrier to $p$-wave electrons results in an apparent activation energy of $0.26 \mathrm{eV}$ (Tossati \& Manini 1994). At high temperatures the sticking coefficients for $C_{60}$ and $C_{70}$ are close to the values given by equation (28).

We adopt equation $(28)$ for $s_{e}(Z=0)$ for silicates as well as for carbonaceous grains, since no laboratory data are available for silicates. In this case, $N_{\mathrm{C}}$ is just a parameter defined by equation (1).

\subsubsection{Attachment to Negatively Charged Grains}

As a grain acquires more electrons, the electron affinity decreases. We take the sticking coefficient to be zero when $Z=Z_{\min }$, since the more negatively-charged state, even if it formed, would autoionize. Thus,

$$
s_{e}(Z<0)=\left\{\begin{array}{ll}
s_{e}(Z=0) & , Z>Z_{\min } \\
0 & , Z \leq Z_{\min }
\end{array} .\right.
$$

\subsubsection{Recombination with Positively Charged Grains}

Electrons arriving at positively charged grains are expected to recombine provided that (1) they do not reflect elastically from the surface and (2) they are able to scatter inelastically before traversing the grain. For small molecules the energy released generally results in dissociation, but for hydrocarbons this most likely results in only the loss of an $\mathrm{H}$ atom, with the carbon skeleton remaining intact. The sticking coefficient is therefore expected to be

$$
s_{e}(Z>0) \approx\left(1-p_{e s}\right)\left(1-e^{-a / l_{e}}\right) \approx 0.5\left(1-e^{-a / l_{e}}\right)
$$

Figure 7 shows measured $s_{e}$ for electron recombination with positive hydrocarbon ions, together with fitting function (30). The measured sticking coefficients generally agree with equation (30) to within about a factor of 2 except for the value measured for naphthalene $\mathrm{C}_{10} \mathrm{H}_{8}^{+}$, which is smaller than predicted by about a factor of 4 . Why this should be smaller is unclear. 


\subsection{Ions}

Ions of interest $\left(\mathrm{H}^{+}\right.$and $\left.\mathrm{C}^{+}\right)$have large ionization potentials, so we assume that they have a high probability of seizing an electron if they arrive at the surface of a grain, whether charged or neutral. While this assumption would be invalid for grains which are already highly positively charged, in practice the rate of arrival of positive ions at the surface of such a grain is in any case negligible. We compute the contribution of ions to the charging rate using equation (26) with $s_{i}=1$.

\section{Ambient Conditions}

\subsection{Radiation Fields}

For some calculations, we adopt a blackbody spectrum for the radiation field, with color temperature $T_{\mathrm{c}}$ and dilution factor $w$, so that $u_{\nu}=4 \pi w B_{\nu}\left(T_{\mathrm{c}}\right) / c$. It is convenient to characterize the radiation intensity by $G \equiv u_{\mathrm{rad}}^{\mathrm{uv}} / u_{\mathrm{Hab}}^{\mathrm{uv}}$, where $u_{\mathrm{rad}}^{\mathrm{uv}}$ is the energy density in the radiation field between $6 \mathrm{eV}$ and $13.6 \mathrm{eV}$ and $u_{\mathrm{Hab}}^{\mathrm{uv}}=5.33 \times 10^{-14} \mathrm{erg} \mathrm{cm}^{-3}$ is the Habing (1968) estimate of the starlight energy density in this range. ${ }^{6}$ The radiation is cut off at $13.6 \mathrm{eV}$.

For the diffuse ISM, we adopt the average interstellar radiation field (ISRF) spectrum in the solar neighborhood, as estimated by Mezger, Mathis, \& Panagia (1982) and Mathis, Mezger, \& Panagia (1983):

$$
\nu u_{\nu}^{\mathrm{ISRF}}=\left\{\begin{array}{ll}
0 & , h \nu>13.6 \mathrm{eV} \\
3.328 \times 10^{-9} \mathrm{erg} \mathrm{cm}^{-3}(h \nu / \mathrm{eV})^{-4.4172} & , 11.2 \mathrm{eV}<h \nu<13.6 \mathrm{eV} \\
8.463 \times 10^{-13} \mathrm{erg} \mathrm{cm}^{-3}(h \nu / \mathrm{eV})^{-1} & , 9.26 \mathrm{eV}<h \nu<11.2 \mathrm{eV} \\
2.055 \times 10^{-14} \mathrm{erg} \mathrm{cm}^{-3}(h \nu / \mathrm{eV})^{0.6678} & , 5.04 \mathrm{eV}<h \nu<9.26 \mathrm{eV} \\
(4 \pi \nu / c) \sum_{i=1}^{3} w_{i} B_{\nu}\left(T_{i}\right) & , h \nu<5.04 \mathrm{eV}
\end{array} ;\right.
$$

the dilution factors $w_{i}$ and blackbody temperatures $T_{i}$ are given in Table 1 . The total energy density in the ISRF of equation (31) is $u=8.64 \times 10^{-13} \mathrm{erg} \mathrm{cm}^{-3}$, with $u_{\mathrm{rad}}^{\mathrm{uv}}=6.07 \times 10^{-14} \mathrm{erg} \mathrm{cm}^{-3}$ in the 6-13.6 eV interval, or $G=1.13$.

The spectrum-averaged absorption efficiency factor is

$$
\left\langle Q_{\mathrm{abs}}\right\rangle \equiv \frac{\int Q_{\mathrm{abs}} u_{\nu} d \nu}{\int u_{\nu} d \nu}
$$

where $Q_{\mathrm{abs}} \pi a^{2}$ is the absorption cross section. In Figure 8 we display $\left\langle Q_{\mathrm{abs}}\right\rangle$ for the ISRF and blackbody spectra with various values of $T_{c}$.

\footnotetext{
${ }^{6}$ For comparison, the interstellar radiation field estimated by Draine (1978) has $u=8.93 \times 10^{-14} \mathrm{erg} \mathrm{cm}^{-3}$ between 6 and $13.6 \mathrm{eV}$, or $G=1.68$.
} 


\subsection{Scaling Law}

The photoelectric emission is dependent on the ambient conditions, which can be characterized by the shape of the radiation spectrum, the gas temperature $T$, and one additional parameter, depending on the ratio $G / n_{e}$, which we take to be $G \sqrt{T} / n_{e}$.

Unless otherwise noted, we will display results for a blackbody spectrum with $T_{\mathrm{c}}=3 \times 10^{4} \mathrm{~K}$, cut off at $13.6 \mathrm{eV}$. In Figure 9, charge distributions are plotted for carbonaceous grains with various sizes in various environments. In Figure 10, the average electrostatic potential, $\langle U\rangle$, is plotted as a function of grain size for two values of $T, 100 \mathrm{~K}$ and $1000 \mathrm{~K}$, and five values of $G \sqrt{T} / n_{e}$, ranging from $10^{2}$ to $10^{6} \mathrm{~K}^{1 / 2} \mathrm{~cm}^{3}$. We also provide results for conditions appropriate for the cold neutral medium (ISRF, $T=100 \mathrm{~K}, n_{e}=0.03 \mathrm{~cm}^{-3}, G \sqrt{T} / n_{e}=380 \mathrm{~cm}^{3} \mathrm{~K}^{1 / 2}$ ) and the warm neutral medium (ISRF, $T=6000 \mathrm{~K}, n_{e}=0.03 \mathrm{~cm}^{-3}, G \sqrt{T} / n_{e}=2900 \mathrm{~cm}^{3} \mathrm{~K}^{1 / 2}$ ). Note that for given $G \sqrt{T} / n_{e}$, the computed potentials show hardly any dependence on $T$.

For low values of $G \sqrt{T} / n_{e}$, the grains can be negatively charged. This is especially so for grains with $a \lesssim l_{e} \approx 10 \AA$, for which the photoemission rate scales approximately with $a^{3}$ rather than $a^{2}$. However, once $a \lesssim 4 \AA$, the potential rapidly rises to positive values; this is a consequence of the rapid decrease in $s_{e}$ for grains with $Z \leq 0$ as $a$ decreases in this range (eq. 28). The wiggles in the curves for high $G \sqrt{T} / n_{e}$ are due to saturation at the positive threshold charge $Z_{I P}$. As the grain size increases, $f_{Z}\left(Z_{I P}\right) \rightarrow 1$, until the size at which $Z_{I P}$ increases by 1 is reached. Thus, although $\langle Z\rangle$ increases monotonically with $a$, it plateaus until $Z_{I P}$ is incremented, so that $\langle U\rangle$ decreases. Once $Z_{I P}$ does increment, $\langle U\rangle$ does not abruptly increase, because the photoemission rate depends on $h \nu-h \nu_{\text {pet }}$ and $h \nu-h \nu_{\text {pdt }}$; thus charge is gradually concentrated in the next higher charge state as $a$ increases.

To see why $G \sqrt{T} / n_{e}$ is a good parameter to describe grain charging, we make the approximation that the charge state on any grain remains constant, i.e.

$$
J_{e}=J_{\mathrm{pe}}+J_{\mathrm{ion}}
$$

We neglect $J_{\text {ion }}$ by virtue of the large proton mass and consider a positively charged grain with $U \sim 1 \mathrm{~V}$. The Draine \& Sutin (1987) expression for $\tilde{J}$ for a grain with static dielectric constant $\epsilon(0) \gg 1$ and $\xi_{i}<0$ is

$$
\tilde{J} \approx\left(1-\frac{\xi_{i}}{\tau_{i}}\right)\left[1+\left(\frac{2}{\tau_{i}-2 \xi_{i}}\right)^{1 / 2}\right],
$$

which expands, for $e U / k T \gg 1$, to give

$$
\tilde{J} \approx \frac{e U}{k T}\left(1+Z^{-1 / 2}\right)
$$

We express

$$
J_{\mathrm{pe}} \propto G\left\langle Q_{\mathrm{abs}}\right\rangle a^{2} g(U, a)
$$


where $\left\langle Q_{\mathrm{abs}}\right\rangle$ is the average value of $Q_{\mathrm{abs}}$ over the range of absorbed photon energies. Here $g(U, a)$ must be a decreasing function of $U$ and $g$ depends on $a$ through the size dependence of the yield $Y$ (see equations 12 and 13). Thus,

$$
\frac{U\left(1+Z^{-1 / 2}\right)}{g(U, a)} \propto\left\langle Q_{\mathrm{abs}}\right\rangle \frac{G \sqrt{T}}{n_{e}}
$$

Note that this result applies only when $G / n_{e}$ is large enough (for given $T$ and $a$ ) to keep the grains strongly positively charged (with $e U \gtrsim k T$ ). For low values of $G / n_{e}, J_{\text {ion }}$ plays a significant role in the grain charging. This introduces an additional dependence on the ionization fraction $x \equiv n_{e} / n_{\mathrm{H}}$, since for the lowest $x$ (i.e. $x \lesssim 2 \times 10^{-4}$ ), the ions are predominantly $\mathrm{C}^{+}$, whereas $\mathrm{H}^{+}$dominates for higher $x$, and $J_{\text {ion }}$ depends on the ion mass. We assume $\mathrm{H}^{+}$in our calculations for the CNM and WNM and $\mathrm{C}^{+}$in our other calculations. For the values of $G \sqrt{T} / n_{e}$ and $T$ considered here, the dependence on ion mix is only significant for the smallest grains, and even then extreme changes in the ion mix lead to only modest changes in the results. In Figure 11 we display $\left(G \sqrt{T} / n_{e}\right)_{0}$, the value of the charging parameter for which $\langle Z\rangle=0$, for carbonaceous and silicate grains, a blackbody radiation field with $T_{\mathrm{c}}=3 \times 10^{4} \mathrm{~K}$, and various gas temperatures. As

$a$ decreases beyond $\approx 4 \AA$ it becomes very difficult for the grains to charge negatively, because $s_{e}$ becomes very small for $Z \leq 0$; thus the curves plunge sharply there.

Incidentally, the presence of $\left\langle Q_{\text {abs }}\right\rangle$ in equation (37) explains the maxima in $\langle U\rangle$ at $a \sim 100 \AA$ (see Figure 10), since $Q_{\text {abs }}$ peaks for $2 \pi a \sim \lambda$, and the photoelectric emission is primarily from radiation with $\lambda \sim 1000 \AA$.

\section{Photoelectric Heating Efficiencies}

The gas heating rate per grain due to photoelectric emission is given by

$$
\Gamma_{\mathrm{pe}}^{\prime}(a)=\sum_{Z} f_{Z}(Z)\left[\Gamma_{\mathrm{pe}, \mathrm{v}}^{\prime}(a)+\Gamma_{\mathrm{pd}}^{\prime}(a)\right]
$$

The contribution from the photoemission of valence electrons is

$$
\Gamma_{\mathrm{pe}, \mathrm{v}}^{\prime}(a)=\pi a^{2} \int_{\nu_{\mathrm{pet}}}^{\nu_{\max }} d \nu Y Q_{\mathrm{abs}} \frac{c u_{\nu}}{h \nu} \int_{E_{\min }}^{E_{\max }} d E f_{E}(E) E
$$

where $E_{\min }=0$ when $Z \geq 0$ and is given by equation (7) when $Z<0$, and $E_{\max }=h \nu-h \nu_{\text {pet }}+E_{\min }$. The photoelectron energy distribution $f_{E}(E)=f_{E}^{0}(E) / y_{2}$ (see eq. 10 and the discussion following it). When $Z<0$, the contribution from photodetachment is given by

$$
\Gamma_{\mathrm{pd}}^{\prime}(a)=\int_{\nu_{\mathrm{pdt}}}^{\nu_{\max }} d \nu \sigma_{\mathrm{pdt}}(\nu) \frac{c u_{\nu}}{h \nu}\left(h \nu-h \nu_{\mathrm{pdt}}+E_{\mathrm{min}}\right) .
$$


We compute the total efficiency for conversion of absorbed radiation into gas heating,

$$
\epsilon_{\Gamma}(a)=\frac{\Gamma_{\mathrm{pe}}^{\prime}(a)-\Lambda_{\mathrm{gr}}^{\prime}(a)}{\pi a^{2} c u_{\mathrm{rad}}\left\langle Q_{\mathrm{abs}}\right\rangle}
$$

Here $\Lambda_{\mathrm{gr}}^{\prime}(a)$ is the rate of energy removal from the gas due to the accretion of charged particles onto the grain, and is given by

$$
\Lambda_{\mathrm{gr}}^{\prime}(a)=\sum_{i} n_{i} s_{i}\left(\frac{8 k T}{\pi m_{i}}\right)^{1 / 2} \pi a^{2} \tilde{\Lambda}\left(\tau_{i}, \xi_{i}\right) k T,
$$

where the sum runs over electrons and ions and $\tau_{i}$ and $\xi_{i}$ are defined below equation (26). Expressions for $\tilde{\Lambda}$ can be found in Draine \& Sutin (1987).

Figures 12 through 14 show results for the gas heating efficiency for the same conditions for which potentials were displayed in Figure 10. The heating efficiency generally decreases as grain size $a$ increases. For low values of $G \sqrt{T} / n_{e}$, this results primarily from the size-dependent yield factor $y_{1}$ (equation 13); the heating efficiency levels off at $a \sim 10^{3} \AA$, where $y_{1}$ levels off. For higher $G \sqrt{T} / n_{e}$, high grain potentials quench the photoemission and result in much lower heating efficiencies; in these cases the smallest grains (with $a \lesssim 100 \AA$ ), with somewhat lower potentials (see Figure 10), dominate the photoelectric heating. The heating efficiencies drop dramatically when $a \lesssim 4 \AA$, as $s_{e}(Z \leq 0)$ decreases rapidly and the grains charge positively.

\section{Net Photoelectric Heating Rate}

In this section we integrate the photoelectric heating efficiency over grain size distributions to find net heating rates. Cardelli, Clayton, \& Mathis (1989) found that the extinction curve and therefore the grain size distribution - varies depending on the environment through which the starlight passes, and that the variation can be roughly parameterized by $R_{V} \equiv A_{V} / E_{B-V}$, the ratio of visual extinction to reddening. For the diffuse ISM, $R_{V} \approx 3.1$; higher values are observed for dense clouds.

Emission in the 3 to $60 \mu \mathrm{m}$ range, presumably generated by grains small enough to reach temperatures of 30 to $600 \mathrm{~K}$ or more upon the absorption of a single starlight photon (see, e.g. Draine $\&$ Anderson 1985), imply a population of very small grains (with $a<50 \AA$ ). The non-detection of the $10 \mu \mathrm{m}$ silicate feature in emission from diffuse clouds (Mattila et al. 1996; Onaka et al. 1996) appears to rule out silicate grains as a major component of the $a \lesssim 15 \AA$ population (Li \& Draine 2000). Emission features at 3.3, 6.2, 7.7, 8.6, and $11.3 \mu \mathrm{m}$ (see Sellgren 1994 for a review) have been identified as $\mathrm{C}-\mathrm{H}$ and $\mathrm{C}-\mathrm{C}$ stretching and bending modes in polycyclic aromatic hydrocarbons (Léger \& Puget 1984), suggesting that the carbonaceous grain population extends down into the molecular regime. The $\mathrm{C}$ abundance ${ }^{7} b_{\mathrm{C}}$ in the very small grain population is still uncertain;

\footnotetext{
${ }^{7}$ By "abundance", we mean the number of atoms of an element per interstellar H nucleus.
} 
comparison of the observed diffuse galactic infrared and microwave emission with detailed model calculations for grains heated by galactic starlight imply $b_{\mathrm{C}} \approx 2-6 \times 10^{-5}$ (Li \& Draine 2000; Draine \& Lazarian 1998a,b; Draine \& Li 2000).

Weingartner \& Draine (2000, hereafter WD00) obtained size distributions with various values of $b_{\mathrm{C}}$ which reproduce the average observed extinction for lines of sight characterized by $R_{V}=3.1$, 4.0, and 5.5. Some of their distributions, designated "case A", were constructed so as to minimize the use of C and Si. For $R_{V}=4.0$ and 5.5, "case B" distributions use the same amount of C and Si as those with $R_{V}=3.1$. Li \& Draine (2000) found that the diffuse galactic infrared emission is best reproduced with the WD00 size distribution with $R_{V}=3.1$ and $b_{\mathrm{C}}=6 \times 10^{-5}$. For this distribution, the $2175 \AA$ hump in the extinction curve is entirely due to the ultrasmall grain population. If this is generally the case throughout the ISM, then we would have $b_{\mathrm{C}}=4 \times 10^{-5}$ when $R_{V}=4.0$ and $b_{\mathrm{C}}=3 \times 10^{-5}$ when $R_{V}=5.5$. We will usually display integrated heating rates computed for the size distributions with these (largest allowed) values of $b_{\mathrm{C}}$.

In Figure 15 we plot the net total gas heating per $\mathrm{H}$ nucleus and per Habing flux,

$$
\frac{\Gamma_{\mathrm{tot}}}{G n_{\mathrm{H}}}=\sum_{c, s} \int_{a_{\min }}^{a_{\max }} \frac{\Gamma_{\mathrm{pe}}^{\prime}-\Lambda_{\mathrm{gr}}^{\prime}}{G} \frac{1}{n_{\mathrm{H}}} \frac{d n_{\mathrm{gr}}}{d a} d a
$$

as a function of $G \sqrt{T} / n_{e}$ for our favored distributions from WD00 with $T=100 \mathrm{~K}$ and $T_{c}=$ $3 \times 10^{4} \mathrm{~K}$. The sum is over the carbonaceous and silicate populations. At a single $G \sqrt{T} / n_{e}$, the heating rates obtained for the several distributions vary by a factor of $\approx 2-4$, showing the sensitivity of $\Gamma_{\text {tot }}$ to the grain size distribution. Of course, the highest heating rates are for the distribution with $R_{V}=3.1$ and $b_{\mathrm{C}}=6 \times 10^{-5}$, which contains the largest population of very small grains. For comparison, we also plot the Bakes \& Tielens (1994) result. When $G \sqrt{T} / n_{e} \gtrsim 10^{4} \mathrm{~K}^{1 / 2} \mathrm{~cm}^{3}$, their curve lies above our $\left(R_{V}=3.1, b_{\mathrm{C}}=6 \times 10^{-5}\right)$ curve, even though they assumed a smaller population of very small grains; this is because they adopted larger electron sticking coefficients than we do. In Figure 16 we show $\Gamma_{\text {tot }} / G n_{\mathrm{H}}$ for dust exposed to the ISRF; here we consider typical diffuse cloud dust with $R_{V}=3.1$, for two possible values of $b_{\mathrm{C}}=0$ and $6 \times 10^{-5}$, and gas temperatures of 100 and $6000 \mathrm{~K}$. Note that the net heating when $T=100 \mathrm{~K}$ does not differ greatly from that displayed in Figure 15 and that the grains have a net cooling effect for low values of $G \sqrt{T} / n_{e}$ when $T=6000 \mathrm{~K}$.

The photoelectric heating rate for the WD00 grain size distributions is fairly well reproduced by the following function:

$$
\Gamma_{\mathrm{pe}} \equiv \sum_{g, s} \int_{a_{\min }}^{a_{\max }} \Gamma_{\mathrm{pe}}^{\prime}(a) \frac{d n_{\mathrm{gr}}}{d a} d a=10^{-26} \frac{\mathrm{erg}}{\mathrm{s}} G n_{\mathrm{H}} \frac{C_{0}+C_{1} T^{C_{4}}}{1+C_{2}\left(G \sqrt{T} / n_{e}\right)^{C_{5}}\left[1+C_{3}\left(G \sqrt{T} / n_{e}\right)^{C_{6}}\right]},
$$

where $T$ is in $\mathrm{K}$ and $G \sqrt{T} / n_{e}$ is in $\mathrm{K}^{1 / 2} \mathrm{~cm}^{3}$. Values for the seven parameters in equation (44) are given in Table 2, along with the largest fractional error, err, for $10 \leq T \leq 10^{4} \mathrm{~K}$ and $10^{2} \leq$ $G \sqrt{T} / n_{e} \leq 10^{6} \mathrm{~K}^{1 / 2} \mathrm{~cm}^{3}$. For most cases shown, we consider a blackbody spectrum with $T_{\mathrm{c}}=$ $3 \times 10^{4} \mathrm{~K}$; we also consider the ISRF for $R_{V}=3.1$. 
Bakes \& Tielens (1994) neglect heating from grains with $a>100 \AA$. We find that this is usually a good approximation; large grains contribute significantly only for low $G \sqrt{T} / n_{e}$ and size distributions with few small grains. This is demonstrated in Table 2, where we give the fraction $h_{s}$ of the total heating due to grains with $a<100 \AA$, for $G \sqrt{T} / n_{e}=10^{2} \mathrm{~K}^{1 / 2} \mathrm{~cm}^{3}$ and $T=100 \mathrm{~K}$. For example, for $R_{V}=3.1, b_{\mathrm{C}}=0.0$, and $T_{\mathrm{c}}=3 \times 10^{4} \mathrm{~K}, 25 \%$ of the heating is contributed by grains with $a>100 \AA$. Bakes \& Tielens also neglect the heating contributed by silicate grains. We find that silicates contribute $\lesssim 25 \%$ of the total heating, with the maximum occuring for the distributions with the fewest very small grains.

The rate of cooling due to charged particle collisions is significant, compared with the photoelectric heating rate, when $T \gtrsim 10^{3} \mathrm{~K}$. The following approximation is fairly accurate when $10^{3} \leq T \leq 10^{4} \mathrm{~K}$ and $10^{2} \leq G \sqrt{T} / n_{e} \leq 10^{6} \mathrm{~K}^{1 / 2} \mathrm{~cm}^{3}$ :

$$
\Lambda_{\mathrm{gr}}=10^{-28} \mathrm{erg} \mathrm{cm}^{3} \mathrm{~s}^{-1} n_{e} n_{\mathrm{H}} T^{\left(D_{0}+D_{1} / x\right)} \exp \left(D_{2}+D_{3} x-D_{4} x^{2}\right),
$$

where $x \equiv \ln \left(G \sqrt{T} / n_{e}\right), T$ is in $\mathrm{K}$, and $G \sqrt{T} / n_{e}$ is in $\mathrm{K}^{1 / 2} \mathrm{~cm}^{3}$. In Table 3 we give the values of $D_{i}$ and the maximum fractional error, err, for the above range of $T$ and $G \sqrt{T} / n_{e}$.

\section{Heating and Cooling in H II Regions}

\subsection{Strömgren Spheres}

Lyman continuum radiation from hot stars photoionizes the surrounding gas, resulting in an $\mathrm{H}$ II region. Studies of the heating in H II regions have found that photoelectric emission from dust can be important, compared with photoionization of H (Maciel \& Pottasch 1982; Oliveira \& Maciel 1986; Maloney, Hollenbach, \& Townes 1992). In these studies, it was assumed that the photoelectric yield $Y$ and absorption efficiency factor $Q_{\text {abs }}$ are independent of grain size and photon energy. Here, we apply our more detailed photoemission model.

In $\mathrm{H}$ II regions, the radiation field includes photons with $h \nu>13.6 \mathrm{eV}$. To see the possible importance of these photons, we calculate integrated photoelectric heating and recombination cooling rates for blackbody spectra with no upper cutoff energy, adopting $T_{\mathrm{c}}=3.5$ and $4.5 \times 10^{4} \mathrm{~K}$. Of course, at any given location in an H II region, there will be a break in the spectrum at $13.6 \mathrm{eV}$, due in part to absorptions along the path to the star and in part to the break in the stellar spectrum itself. Thus, spectra with no break and with a cutoff at $13.6 \mathrm{eV}$ should bracket the range applicable in $\mathrm{H}$ II regions.

We take $n_{e} / n_{\mathrm{H}}=1$ and $T=9000 \mathrm{~K}$ for the gas. In order to estimate likely $G / n_{\mathrm{H}}$ values, we consider an $\mathrm{O} 9 \mathrm{~V}$ exciting star and a point at the half-mass radius (i.e. at the distance from the star for which half of the H II region volume is enclosed). The radius of the H II region (the 
"Strömgren radius") is found by balancing ionizations against recombinations:

$$
R_{S}=\left(\frac{3}{4 \pi} \frac{\dot{N}_{\mathrm{Ly}}}{\alpha n_{\mathrm{H}}^{2}}\right)^{1 / 3},
$$

where $\dot{N}_{\text {Ly }}$ is the rate at which the star produces ionizing photons and the case B recombination coefficient $\alpha \approx 2.6 \times 10^{-13} T_{4}^{-0.8} \mathrm{~cm}^{3} \mathrm{~s}^{-1}$, where $T_{4} \equiv T / 10^{4} \mathrm{~K}$ (Osterbrock 1989). Since $R_{S} \propto n_{\mathrm{H}}^{-2 / 3}$, $G / n_{\mathrm{H}} \propto n_{\mathrm{H}}^{1 / 3}$. We take $\dot{N}_{\mathrm{Ly}}=3.63 \times 10^{48} \mathrm{~s}^{-1}$, luminosity $L=4.4 \times 10^{38} \mathrm{erg} \mathrm{s}^{-1}$, and effective temperature $T_{\text {eff }}=3.59 \times 10^{4} \mathrm{~K}$ (Vacca, Garmany, \& Shull 1996). For $n_{\mathrm{H}}=0.1\left(10^{3}\right) \mathrm{cm}^{-3}$, $G / n_{\mathrm{H}} \approx 0.5(10.) \mathrm{cm}^{3}$.

In Tables 4 and 5 , we give $\Gamma_{\mathrm{pe}} / G n_{\mathrm{H}}$ and $\Lambda_{\mathrm{gr}} / G n_{\mathrm{H}}$ for $G / n_{\mathrm{H}}=0.1,1$, and $10 \mathrm{~cm}^{3}$, both with and without the cutoff at $13.6 \mathrm{eV}$, for several of the WD00 grain size distributions. The heating rate typically increases by a factor of a few when the cutoff is removed, and the cooling rate is modestly affected, due to changes in the charging. When $G / n_{\mathrm{H}}=1.0 \mathrm{~cm}^{3}$, grains have a net cooling (heating) effect for radiation with(out) a cutoff at $13.6 \mathrm{eV}$; grains always have a net heating effect when $G / n_{\mathrm{H}}=10 . \mathrm{cm}^{3}$. When $G / n_{\mathrm{H}}<0.1 \mathrm{~cm}^{3}$ the grains are negatively charged, so that $\Gamma_{\mathrm{pe}} / G n_{\mathrm{H}} \approx$ constant, and $\Lambda_{\mathrm{gr}} / G n_{\mathrm{H}} \propto\left(G / n_{\mathrm{H}}\right)^{-1}$, roughly.

The net steady-state heating rate due to photoionization is, in the notation of Spitzer (1978),

$$
\Gamma_{\mathrm{pi}} \approx \alpha n_{e}^{2}\left(\langle\psi\rangle k T_{\mathrm{c}}-\left(\chi_{2} / \phi_{2}\right) k T\right) \approx 1.5 \times 10^{-24} n_{e}^{2} T_{4}^{-0.8}\left(\frac{\langle\psi\rangle T_{\mathrm{c}}-\left(\chi_{2} / \phi_{2}\right) T}{42000 \mathrm{~K}}\right) \mathrm{erg} \mathrm{cm}^{3} \mathrm{~s}^{-1}
$$

where $\langle\psi\rangle k T_{\mathrm{c}}$ is the mean kinetic energy per photoelectron, and $\left(\chi_{2} / \phi_{2}\right) k T$ is the mean kinetic energy per recombining electron for case B recombination; $\langle\psi\rangle \approx 1.067\left(T_{\mathrm{c}} / 10^{4} \mathrm{~K}\right)^{0.23}$ for $16000 \lesssim$ $T_{\mathrm{c}} \lesssim 64000 \mathrm{~K}$, and $\chi_{2} / \phi_{2} \approx 0.67 T_{4}^{-0.13}$ (Spitzer 1978). Comparing with the entries in Tables 4 and 5 , we find that the net heating from dust can be comparable to or exceed the photoionization heating when $G / n_{\mathrm{H}} \gtrsim 5 \mathrm{~cm}^{3}$.

In an actual H II region, the grain size distribution could be a function of distance from the exciting star, and could differ substantially from those found by WD00. The very small grains might be destroyed in the harsh ionizing environment and the large grains might drift away from the star. A detailed study of the contribution of photoelectric heating in H II regions would have to account for these effects.

\subsection{The Warm Ionized Medium}

The warm ionized medium (WIM), also known as the diffuse ionized gas (DIG), extends to distances $|z|>1 \mathrm{kpc}$ above the Galactic midplane (see Reynolds 1990, 1993 for reviews). It is not yet clear how the WIM is ionized and heated. The only known source of ionization with adequate power to maintain the WIM is Lyman continuum radiation from O stars, but it is not clear how the ionizing photons can reach locations with high $|z|$, since atomic gas is highly opaque to these 
photons. Observed emission line intensity ratios can be approximately reproduced by models in which photoionization by a dilute radiation field dominates (Domgörgen \& Mathis 1994). However, such models fail to account for observed variations in line intensity ratios with $|z|$ (see Reynolds, Haffner, \& Tufte 1999 and references therein).

Haffner, Reynolds, \& Tufte (1999) found that the line intensity variations could be due to variations in the gas temperature; specifically, they suggested an increase from $T \approx 7000 \mathrm{~K}$ at $|z| \approx 500 \mathrm{pc}$ to $T \approx 10^{4} \mathrm{~K}$ at $|z| \approx 1500 \mathrm{pc}$. Reynolds et al. (1999) noted that the variations are more generally correlated with the electron density, suggesting a supplemental heat source that dominates photoionization heating at low densities. They found that a supplemental heat source $\Gamma \sim 10^{-25} n_{\mathrm{H}} \operatorname{erg~s}^{-1}$ would result in a temperature profile $T(|z|)$ which could account for the observed line intensity ratio variations, and suggested photoelectric heating by dust as a possibility.

In Figure 17, we plot the $T(|z|)$ profile inferred by Reynolds et al. (1999) from observed values of the $[\mathrm{N} \mathrm{II}] / \mathrm{H} \alpha$ line intensity ratio. In this section we will investigate whether the combination of heating by photoionization and photoemission from dust can reproduce this profile. Reynolds et al. find that the electron density profile can be approximated by

$$
n_{e}(|z|)=0.125 T_{4}^{0.45} f^{-0.5} \exp (-|z| / 1 \mathrm{kpc}) \mathrm{cm}^{-3},
$$

where $T_{4}=T / 10^{4} \mathrm{~K}$ and $f$ is the WIM volume filling fraction. Reynolds et al. considered two cases for $f(|z|): f(|z|)=0.2$ and $f(|z|)=0.1 \exp (|z| / 0.75 \mathrm{kpc}$ ) (Kulkarni \& Heiles 1987). We calculate temperature profiles for four different cases (A-D), as summarized in Table 6, employing the above two prescriptions for the filling factor and two grain size distributions, for $R_{V}=3.1$ and $b_{\mathrm{C}}=0$ and $6 \times 10^{-5}$. We take the net heating rate due to photoionization $\Gamma_{\mathrm{pi}}=1.5 \times 10^{-24} n_{e}^{2} T_{4}^{-0.8} \mathrm{erg} \mathrm{cm}^{3} \mathrm{~s}^{-1}$ (appropriate for $T_{\mathrm{c}} \approx 3.5 \times 10^{4} \mathrm{~K}$; equation 47 ) and cooling rate (excluding grain collisional cooling) $\Lambda_{\text {gas }}=3.0 \times 10^{-24} T_{4}^{1.9} n_{e}^{2} \mathrm{erg} \mathrm{cm}^{3} \mathrm{~s}^{-1}$, which approximates the cooling function for ionized gas given in Figure 3.2 of Osterbrock (1989), and find $T$ satisfying

$$
\Gamma_{\mathrm{pi}}+\Gamma_{\mathrm{pe}}=\Lambda_{\mathrm{gas}}+\Lambda_{\mathrm{gr}}
$$

We plot the temperature profiles for the four cases A-D in Figure 17; we have adjusted the values of $G$ so that the curves roughly lie on top of the Reynolds et al. curve. In Figure 18, we plot the associated values of the charging parameter $G \sqrt{T} / n_{e}$ and the net grain heating $\Gamma_{\text {tot }} / n_{\mathrm{H}}$ as functions of $|z|$; note that $\Gamma_{\text {tot }} / n_{\mathrm{H}}$ is not constant, since $T$ and $G \sqrt{T} / n_{e}$ vary with $|z|$. For each of the cases $\mathrm{A}-\mathrm{D}$, the temperature profiles are too shallow compared with the Reynolds et al. curve. One might expect steeper curves to result if $G$ is increased, although this might require reducing $\Gamma_{\mathrm{pi}} / n_{e}^{2}$ to unreasonably small values. However, this is not usually the case, because the photoelectric heating efficiency begins to drop rapidly when $G \sqrt{T} / n_{e} \gtrsim 10^{3} \mathrm{~K}^{1 / 2} \mathrm{~cm}^{3}$. We can obtain a reasonable match to the Reynolds et al. curve by altering case $\mathrm{D}$ somewhat, so that $\Gamma_{\mathrm{pi}}=0.7 \times 10^{-24} n_{e}^{2} T_{4}^{-0.8} \mathrm{erg} \mathrm{cm}^{3} \mathrm{~s}^{-1}$ and $G=0.27$ (case E). ${ }^{8}$ Thus, we can reproduce the Reynolds

\footnotetext{
${ }^{8}$ This could arise, for example, for $T_{\mathrm{c}} \approx 26000 \mathrm{~K}$.
} 
et al. temperature profile by adopting a large population of very small grains and the Kulkarni \& Heiles (1987) formula for the WIM filling factor as a function of $|z|$. Other heating mechanisms, such as the dissipation of interstellar turbulence (Minter \& Spangler 1997), could also contribute, but do not appear to be required to account for the observed temperatures.

\section{Summary}

We have presented a model for photoelectric emission from dust. Here we first summarize how our model differs from the recent study by Bakes \& Tielens (1994) and the uncertainties that remain.

We have re-evalulated the photoemission threshold energies for small grains, based in part on an empirical approach employing laboratory data for ionization potentials and electron affinities of PAH molecules. For negatively charged grains, we consider both photoemission from the valence band and the removal of attached electrons, and set the photoemission and photodetachment threshold energies equal to the minimum excitation energy for which an electron can effectively tunnel across the Coulomb barrier. Bakes \& Tielens take the emission threshold equal to the electron affinity, which is an underestimate. Motivated by the PAH hypothesis, Bakes \& Tielens adopt a disk geometry for the smallest grains. The geometry affects the ionization potential through the capacitance, but this effect is small. We assume spherical grains for simplicity, and thereby retain consistency with the calculation of the charged particle accretion rates $J_{e}$ and $J_{\text {ionn }}$.

We treat the variation of the photoelectric yield with grain charge and the distribution of photoelectron energies in a consistent manner (eq. 11). Whereas Bakes \& Tielens adopt a delta function for the distribution of photoelectron energies, we adopt a parabolic form (eq. 10). We adopt a yield function for carbonaceous grains which, like that of Bakes \& Tielens, approximately reproduces the measured ionization yield of coronene for grains with $a \approx 4 \AA$, but which agrees better with experimental determinations of the bulk graphite yield. Still, our bulk yields substantially exceed the experimental results; see the discussion following equation (16). In computing the enhancement factor for the yield of small particles, we evaluate the photon attenuation length $l_{a}$ as a function of wavelength (eq. 14), rather than adopting $l_{a}=100 \AA$, independent of wavelength, as Bakes \& Tielens do. Recall, however, that the expression for the size-dependent yield enhancement (eq. 13) is based on a simple theoretical model; laboratory investigation of photoemission from microscopic particles is still in its infancy.

Recent experimental results have been used to obtain new estimates for electron sticking efficiencies, as a function of grain size, shown in Figures 6 and 7. We have applied our photoemission and collisional charging model to compute the efficiency with which grains convert absorbed radiation into gas heating via the photoelectric effect, as a function of grain size ( $\S 5$, Figures 12 - 14). Bakes \& Tielens (1994) derived photoelectric heating rates for H I regions by integrating their heating efficiencies over an MRN size distribution with the lower cutoff size for the graphite 
population extended to $3.5 \AA$. We integrate the net gas heating rate over the size distributions of Weingartner \& Draine (2000) (Figures 15 and 16) and we provide fitting functions for the resulting total photoelectric heating and recombination cooling rates ( 66$)$. In a separate paper, we will examine the consequences of these revised rates for the thermal structure of the ISM.

Finally, we have applied our model to estimate rates for photoelectric heating by dust grains in $\mathrm{H}$ II regions $(\S 7)$. We find that photoelectric heating by dust can be important in $\mathrm{H}$ II regions when $G / n_{\mathrm{H}} \gtrsim 1 \mathrm{~cm}^{3}$. Photoelectric heating in the warm ionized medium (WIM) might explain the observed variations in emission line intensity ratios with distance from the Galactic midplane, if the abundance of ultrasmall grains is large enough. The required abundance is equal to that adopted by Li \& Draine (2000) to account for the diffuse galactic infrared emission.

We have investigated a range of grain sizes, gas temperatures, radiation field color temperatures, and ratios $G / n_{e}$ of radiation intensity to electron density, and selected results have been presented. Interested readers can find a FORTRAN routine that implements the heating and cooling approximations of equations (44) and (45) on the World Wide Web at www.cita.utoronto.ca/ weingart.

This research was supported in part by NSF grant AST-9619429 and by NSF Graduate and International Research Fellowships to JCW. We are grateful to P. A. M. van Hoof for helpful comments on the manuscript, L. M. Haffner for providing us with an electronic version of the Reynolds et al. (1999) $T(z)$ data, and R. H. Lupton for the availability of the SM plotting package.

\section{REFERENCES}

Abouelaziz, H., Gomet, J. C., Pasquerault, D., Rowe, B. R., \& Mitchell, J. B. A. 1993, J. Phys. Chem., 99, 237

Allamandola, L. J., Tielens, A. G. G. M., \& Barker, J. R. 1989, ApJS, 71, 733

Bakes, E. L. O. 1992, Ph.D. thesis, Univ. of London

Bakes, E. L. O., \& Tielens, A. G. G. M. 1994, ApJ, 427, 822

Bohren, C. F. \& Huffman, D. R. 1983, Absorption and Scattering of Light by Small Particles (New York: Wiley)

Burtscher, H. \& Schmidt-Ott, A. 1982, Phys. Rev. Lett., 48, 1374

Burtscher, H., Schmidt-Ott, A., \& Siegmann, H.C. 1984, Z. Phys. B, 56, 197

Canosa, A., Parent, D. C., Pasquerault, D., Gomet, J. C., Laubé, S., \& Rowe, B. R. 1994, Chem. Phys. Let., 228, 26

Cardelli, J. A., Clayton, G. C., \& Mathis, J. S. 1989 (CCM), ApJ, 345, 245 
Chen, G. D., Ma, S. G., Cooks, R. G., Bronstein, H. E., Best, M. D., \& Scott, L. T. 1997, J. Mass. Spectrom., 32, 1305

Chen, E. S., Chen, E. C. M., Sane, N., Talley, L., Kozanecki, N., \& Shulze, S. 1999, J. Chem. Phys., 110,9319

Christophorou, L. G., Datskos, P. G., \& Faidas, H. 1994, J. Chem. Phys., 101, 6728

Dartois, E., \& d'Hendecourt, L. 1997, A\&A, 323, 534

de Jong, T. 1977, A\&A, 55, 137

Domgörgen, H. \& Mathis, J. S. 1994, ApJ, 428, 647

Draine, B. T. 1978, ApJS, 36, 595

Draine, B. T. \& Anderson, N. 1985, ApJ, 292, 494

Draine, B. T. \& Lazarian, A. 1998a, ApJ, 494, L19

Draine, B. T. \& Lazarian, A. 1998b, ApJ, 508, 157

Draine, B. T. \& Lee, H. M. 1984, ApJ, 285, 89

Draine, B. T. \& Li, A. 2000, in preparation

Draine, B. T., \& Salpeter 1979, ApJ, 231, 77

Draine, B. T., \& Sutin, B. 1987, ApJ, 320, 803

Dwek, E. \& Smith, R. K. 1996, ApJ, 459, 686

Faraci, G., Pennisi, A. R., \& Margaritondo, G. 1989, Phys. Rev. B, 40, 4209

Feuerbacher, B., Anderegg, M., Fitton, B., Laude, L. D., Willis, R. F., \& Grard, R. J. L. 1972, Geochim. Cosmochim. Acta Suppl. 2, 3, 2655

Feuerbacher, B. \& Fitton, B. 1972, J. Appl. Phys, 43, 1563

Fujihira, M., Hirooka, R., \& Inokuchi, H. 1973, Chem. Phys. Lett., 19, 584

Habing, H. J. 1968, Bull. Astron. Inst. Netherlands, 19, 421

Haffner, L. M., Reynolds, R. J., \& Tufte, S. L. 1999, ApJ, 523, 223

Huffman, D. R. \& Stapp, J. L. 1973, in IAU Symposium 52, Interstellar Dust and Related Topics, ed. J. M. Greenberg \& H. C. van de Hulst (Dordrecht: Reidel), 297

Kim, S.-H. \& Martin, P. G. 1995, ApJ, 442, 172 
Kulkarni, S. \& Heiles C. 1987, in Interstellar Processes, ed. D. J. Hollenbach \& H. A. Thronson, Jr. (Dordrecht: Reidel), 87

Lang, N. D. \& Kohn, W. 1971, Phys. Rev. B, 3, 1215

Laor, A. \& Draine, B. T. 1993, ApJ, 402, 441

Léger, A. \& Puget, J. L. 1984, A\&A, 137, L5

Lehfaoui, L, Rebrion-Rowe, C., Laubé, S., Mitchell, J. B. A., \& Rowe, B. R. 1997, J. Chem. Phys., 106,5406

Li, A. \& Draine, B. T. 2000, in preparation

Lias, S. G., et al. 1988, J. Phys. Chem. Ref. Data, 17, Suppl. 1

Lichtenberger, D. L., Nebesny, K. W., Ray, C. D., Huffman, D. R., \& Lamb, L. D. 1991, Chem. Phys. Lett., 176, 203

Lichtenberger, D. L., Rempe, M. E., \& Gogosha, S. B. 1992, Chem. Phys. Lett., 198, 454

Maciel, W. J. \& Pottasch, S. R. 1992, A\&A, 106, 1

Makov, G., Nitsan, N., \& Brus, L. E. 1988, J. Chem. Phys., 88, 5076

Maloney, P. R., Hollenbach, D. J., \& Townes, C. H. 1992, ApJ, 401, 559

Martin, C., Arakawa, E. T., Callcott, T. A., \& Warmack, R. J. 1987, J. Electr. Spectrosc. Rel. Phenom., 42, 171

Mathis, J.S., Mezger, P.G., \& Panagia, N. 1983, A\&A, 128, 212

Mattila, K., Lemke, D., Haikala, L. K., Laureijs, R. J., Léger, A., Lehtinen, K., Leinert, Ch., \& Mezger, P. G. 1996, A\&A, 315, L353

McFeely, F. R., Cartier, E., Yarmoff, J. A., \& Joyce, S. A. 1990, Phys. Rev. B, 42, 5191

Mezger, P.G., Mathis, J.S., \& Panagia, N. 1982, A\&A, 105, 372

Minter, A. H. \& Spangler, S. R. 1997, ApJ, 485, 182

Moskovits, M. 1991, Ann. Rev. Phys. Chem., 42, 465

Moustefaoui, T., Rebrione-Rowe, C., Le Garrec, J.-L., Rowe, B. R., \& Mitchell, J. B. A. 1998, Faraday Discuss., 109, 71

Müller, U., Schmidt-Ott, A., \& Burtscher, H. 1988, Z. Phys. B, 73, 103

Nayak, S. K., Rao, B. K., Khanna, S. N., \& Jena, P. 1998, J. Chem. Phys., 109, 1245 
O’Donnell, J. E. 1994, ApJ, 422, 158

Oliveira, S. \& Maciel, W. J. 1986, Ap\&SS, 126, 211

Onaka, T., Yamamura, I., Tanabé, T., Roellig, T. L., \& Yuen, L. 1996, PASJ, 48, L59

Osterbrock, D. E. 1989, Astrophysics of Gaseous Nebulae and Active Galactic Nuclei (Mill Valley: University Science Books)

Rebrion-Rowe, C., Lehfaoui, L., Rowe, B. R., \& Mitchell, J. B. A. 1998, J. Chem. Phys., 108, 7185

Reynolds, R. J. 1990, in IAU Symp. 144, The Interstellar Disk/Halo Connection in Galaxies, ed. H. Bloemen (Dordrecht: Kluwer), 67

Reynolds, R. J. 1993, in Back to the Galaxy, ed. S. S. Holt \& F. Verter (A.I.P. Conf. Proc. 278), 156

Reynolds, R. J., Haffner, L. M., \& Tufte, S. L., ApJ, 525, L21

Rowe, B. R., Canosa, A., \& Le Page, V. 1995, Int. J. Mass Spectroscopy, 149, 573

Ruoff, , R. S., Kadish, R. M., Boulas, P., \& Chen, E. C. M. 1995, J. Phys. Chem., 99, 8843

Schiedt, J., \& Weinkauf, R. 1997, Chem. Phys. Lett., 266, 201

Schmidt-Ott, A., Schurtenberger, P., \& Siegmann, H. C. 1980, Phys. Rev. Lett., 45, 1284

Seidl, M. \& Brack, M. 1996, Annals of Physics, 245, 275

Sellgren, K., 1994, in The Infrared Cirrus and Diffuse Interstellar Clouds, ed. R. Cutri \& W. B. Latter, A.S.P. Conference Series, 58, 243

Smith, D., Spanel, P., \& Märk, T. D. 1993, Chem. Phys. Lett., 213, 202

Spain, I. L. 1973, Chemistry and Physics of Carbon, 8, 1

Spanel, P., \& Smith, D. 1994, Chem. Phys. Lett., 229, 262

Spitzer, L., Jr. 1948, ApJ, 107, 6

Spitzer, L., Jr. 1978, Physical Processes in the Interstellar Medium (New York: Wiley)

Steger, H., DeVries, J., Kamke, B., Kamke, W., Drewello, T. 1992, Chem. Phys. Lett., 194, 452

Tielens, A. G. G. M. 1993, in Dust and Chemistry in Astronomy, eds. T. J. Millar \& D. A. Williams, (Bristol: Inst. of Physics), 103

Tielens, A. G. G. M., \& Hollenbach, D. J. 1985, ApJ, 291, 722 
Tobita, S., Leach, S., Jochims, H. W., Rühl, E., Illenberger, E., \& Baumgärtel, H. 1994, Can. J. Phys., 72, 1060

Tobita, S., Meinke, M., Illenberger, E., Christophourou, L. G., Baumgärtel, H., \& Leach, S. 1992, Chem. Phys. 161, 501

Tosatti, E., \& Manini, N. 1994, Chem. Phys. Lett. 223, 61

Vacca, W. D., Garmany, C. D., \& Shull, J. M. 1996, ApJ, 460, 914

Verstraete, L., Léger, A., d'Hendecourt, L., Dutuit, O., \& Défourneau, D. 1990, A\&A, 237, 436

Wang, L.-S., Desai, S. R., Wu, H., \& Nichloas J. B., 1997, Z. Phys. D, 40, 36

Wang, X.B., Ding, C.F., \& Wang, C.F. 1999, J. Chem. Phys., 110, 8217

Watson, W. D. 1972, ApJ, 176, 103

Watson, W. D. 1973, J. Opt. Soc. Am., 63, 164

Weingartner, J. C. \& Draine, B. T. 2000, submitted, astro-ph/0008146 (WD00) 

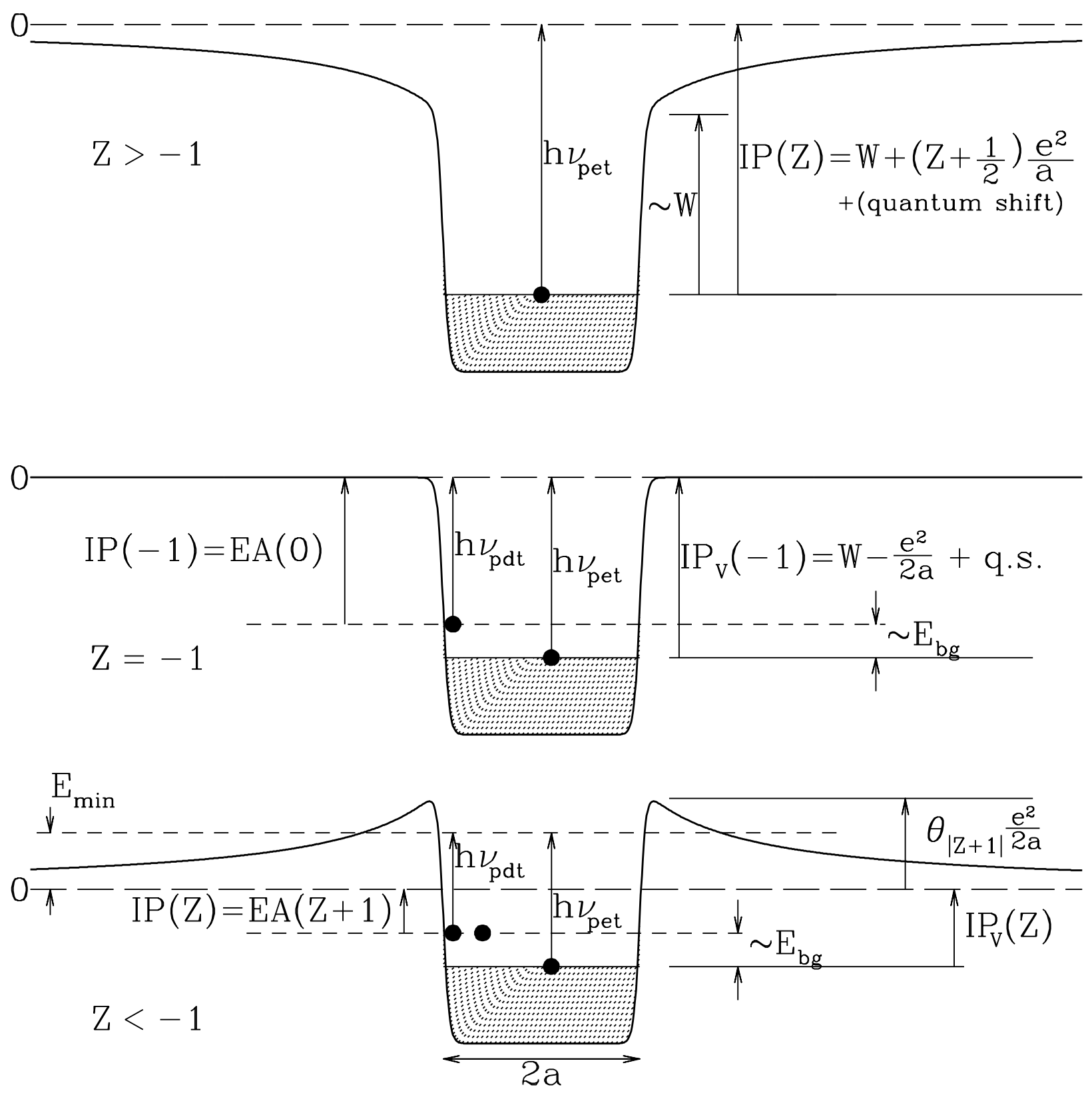

Fig. 1. - The potential confining electrons in a grain with charge Ze. Shaded regions show occupied energy levels and $W$ is the work function. Upper panel: $Z>-1$. The photoemission threshold photon energy $h \nu_{\text {pet }}=I P$, the ionization potential. Middle panel: $Z=-1$. The extra electron occupies the lowest unoccupied energy level (LUMO) of the neutral grain, lying a distance $E A(Z=0)$ below zero (EA is the electron affinity). The ionization potential $\operatorname{IP}(Z=-1)$ and photodetachment threshold energy $h \nu_{\text {pdt }}$ both equal $E A(Z=0)$. The photoemission threshold energy $h \nu_{\text {pet }}=I P_{V}$, the valence band ionization potential. $I P_{V}-I P \approx E_{\mathrm{bg}}$, the energy band gap in bulk material; the equality is not exact due to quantum shifts in energy levels with grain size. Lower panel: $Z<-1$. When an electron acquires an energy $E_{\min }$ above zero, it can tunnel out of the grain. Thus, $h \nu_{\mathrm{pdt}}=I P(Z)+E_{\min }$ and $h \nu_{\mathrm{pet}}=I P_{V}(Z)+E_{\min }$. When $Z<-1$, the maximum value of the confining potential is $\theta_{|Z+1|} e^{2} / a$; the parameter $\theta_{|Z+1|}$ is derived by Draine \& Sutin (1987). 

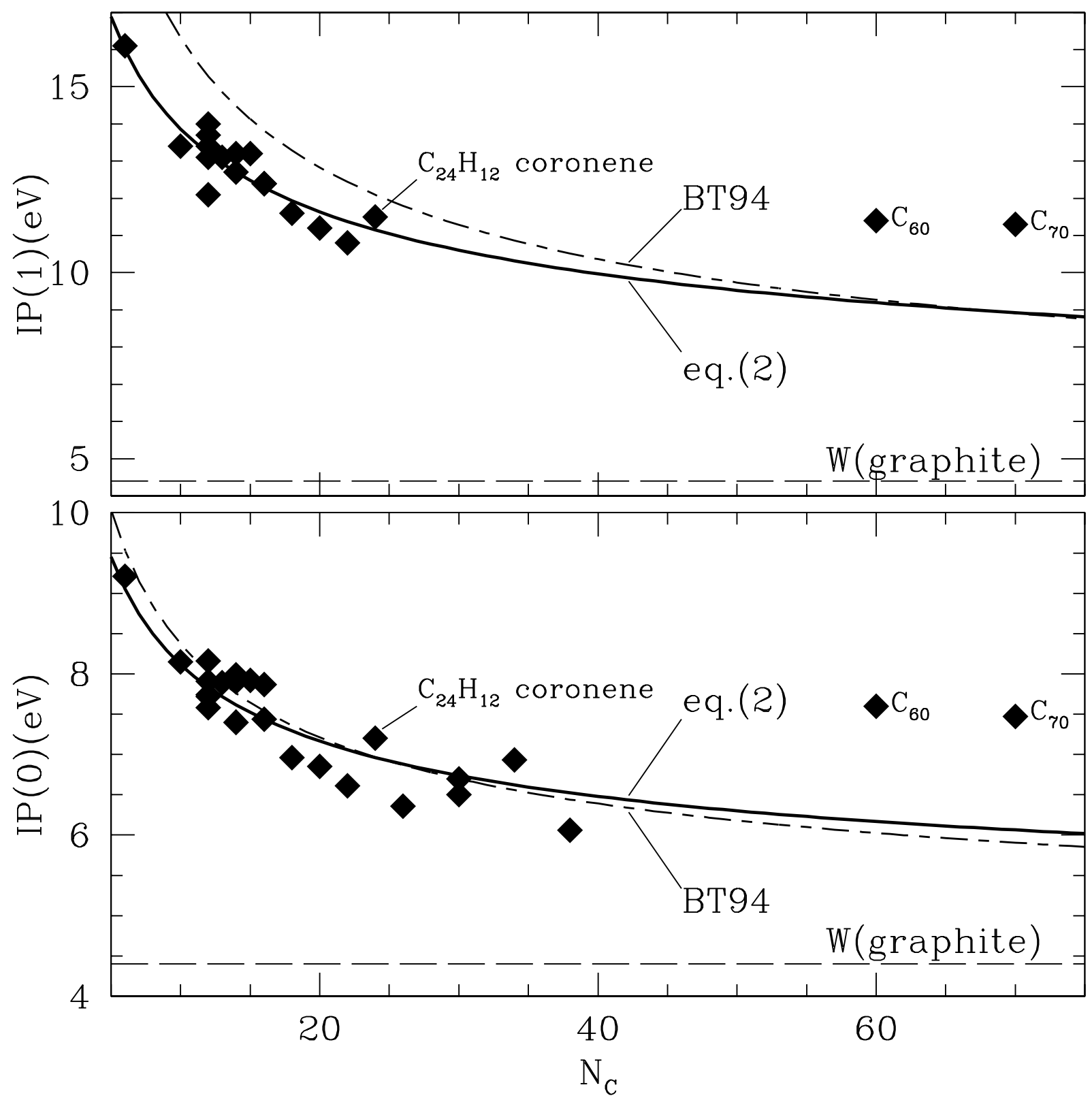

Fig. 2.- First and second ionization potentials $I P(0)$ and $I P(1)$ for cyclic aromatic hydrocarbons and fullerenes. Solid curve is eq.(2). Curve labelled BT94 is the IP estimate of Bakes \& Tielens (1994). Data: Lias et al. (1988) for $\mathrm{C}_{14} \mathrm{H}_{10}$ anthracene, $\mathrm{C}_{26} \mathrm{H}_{16}$ hexacene, $\mathrm{C}_{30} \mathrm{H}_{14}$ dibenz[bc,hl]-coronene, $\mathrm{C}_{30} \mathrm{H}_{16}$ pyranthene, $\mathrm{C}_{34} \mathrm{H}_{18}$ tetrabenz[a,cdj,lm]-perylene; Tobita et al. (1994) for $\mathrm{C}_{10} \mathrm{H}_{8}$ naphthalene, $\mathrm{C}_{12} \mathrm{H}_{8}$ biphenylene, $\mathrm{C}_{12} \mathrm{H}_{8}$ acenaphthlylene, $\mathrm{C}_{12} \mathrm{H}_{10}$ biphenyl, $\mathrm{C}_{12} \mathrm{H}_{10}$ acenaphthene, $\mathrm{C}_{12} \mathrm{H}_{10}$ 2-vinylnaphthalene, $\mathrm{C}_{13} \mathrm{H}_{10}$ fluorene, $\mathrm{C}_{14} \mathrm{H}_{10}$ diphenylacetylene, $\mathrm{C}_{14} \mathrm{H}_{12}$ 9,10-dihydrophenanthrene, $\mathrm{C}_{14} \mathrm{H}_{9} \mathrm{~N}$ acridine, $\mathrm{C}_{16} \mathrm{H}_{10}$ pyrene $\mathrm{C}_{16} \mathrm{H}_{10}$ fluoranthene, $\mathrm{C}_{18} \mathrm{H}_{12}$ tetracene, $\mathrm{C}_{20} \mathrm{H}_{12}$ perylene, $\mathrm{C}_{22} \mathrm{H}_{14}$ pentacene, $\mathrm{C}_{24} \mathrm{H}_{12}$ coronene; Chen et al. (1999) for $\mathrm{C}_{38} \mathrm{H}_{20}$ benz[42]; Lichtenberger et al. (1991), Lichtenberger et al. (1992), and Steger et al. (1992) for $\mathrm{C}_{60}$, $\mathrm{C}_{70}$ fullerenes. 


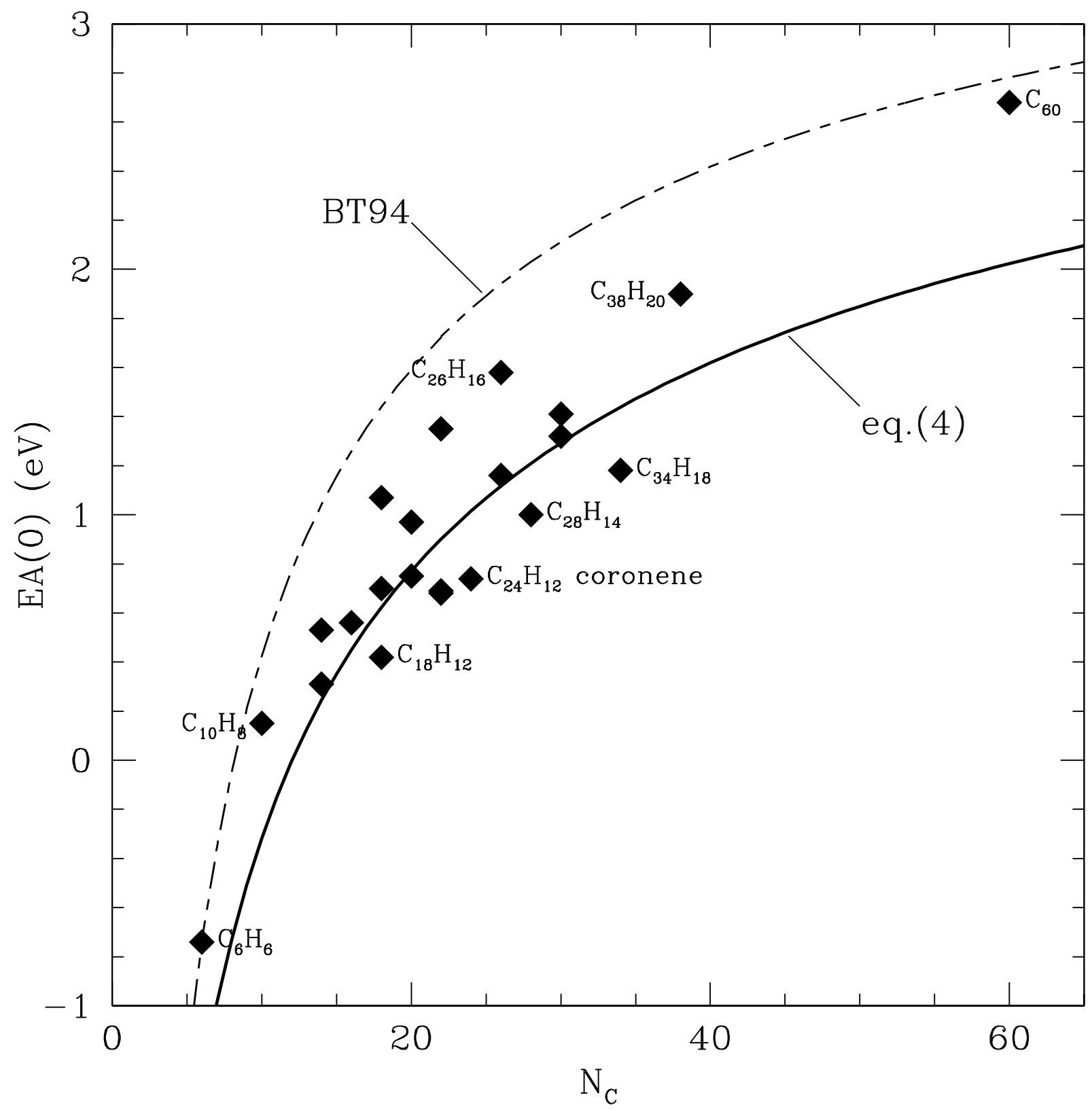

Fig. 3.- Electron affinity for neutral PAH molecules and $\mathrm{C}_{60}$. Solid curve is eq.(4). Curve labelled BT94 is estimate of Bakes (1992), used by Bakes \& Tielens (1994). Data: Chen et al. (1999) for $\mathrm{C}_{6} \mathrm{H}_{6}$ benzene, $\mathrm{C}_{24} \mathrm{H}_{12}$ coronene, $\mathrm{C}_{26} \mathrm{H}_{16}$ hexacene, $\mathrm{C}_{30} \mathrm{H}_{14}$ dibenz[bc,hl]-coronene, $\mathrm{C}_{30} \mathrm{H}_{16}$ pyranthene, $\mathrm{C}_{34} \mathrm{H}_{18}$ tetrabenz[a,cdj,lm]-perylene, $\mathrm{C}_{38} \mathrm{H}_{20}$ benz[42]; Shiedt \& Weinkauf (1997) for $\mathrm{C}_{14} \mathrm{H}_{10}$ anthracene; Ruoff et al. (1995) for $\mathrm{C}_{10} \mathrm{H}_{8}$ naphthalene, $\mathrm{C}_{14} \mathrm{H}_{10}$ phenanthrene, $\mathrm{C}_{16} \mathrm{H}_{10}$ pyrene, $\mathrm{C}_{18} \mathrm{H}_{12}$ tetracene, $\mathrm{C}_{18} \mathrm{H}_{12}$ benzanthracene, $\mathrm{C}_{18} \mathrm{H}_{12}$ chrysene, $\mathrm{C}_{20} \mathrm{H}_{12}$ perylene, $\mathrm{C}_{20} \mathrm{H}_{12}$ benzo(a)pyrene, $\mathrm{C}_{22} \mathrm{H}_{14}$ pentacene, $\mathrm{C}_{22} \mathrm{H}_{14}$ dibenz(a,j)anthracene; Chen et al. (1997) for $\mathrm{C}_{26} \mathrm{H}_{12}$ diindenochrysene, $\mathrm{C}_{28} \mathrm{H}_{14}$ debenzo[a,g]corannulene; and Wang, Ding, \& Wang (1999) for $\mathrm{C}_{60}$ fullerene. 


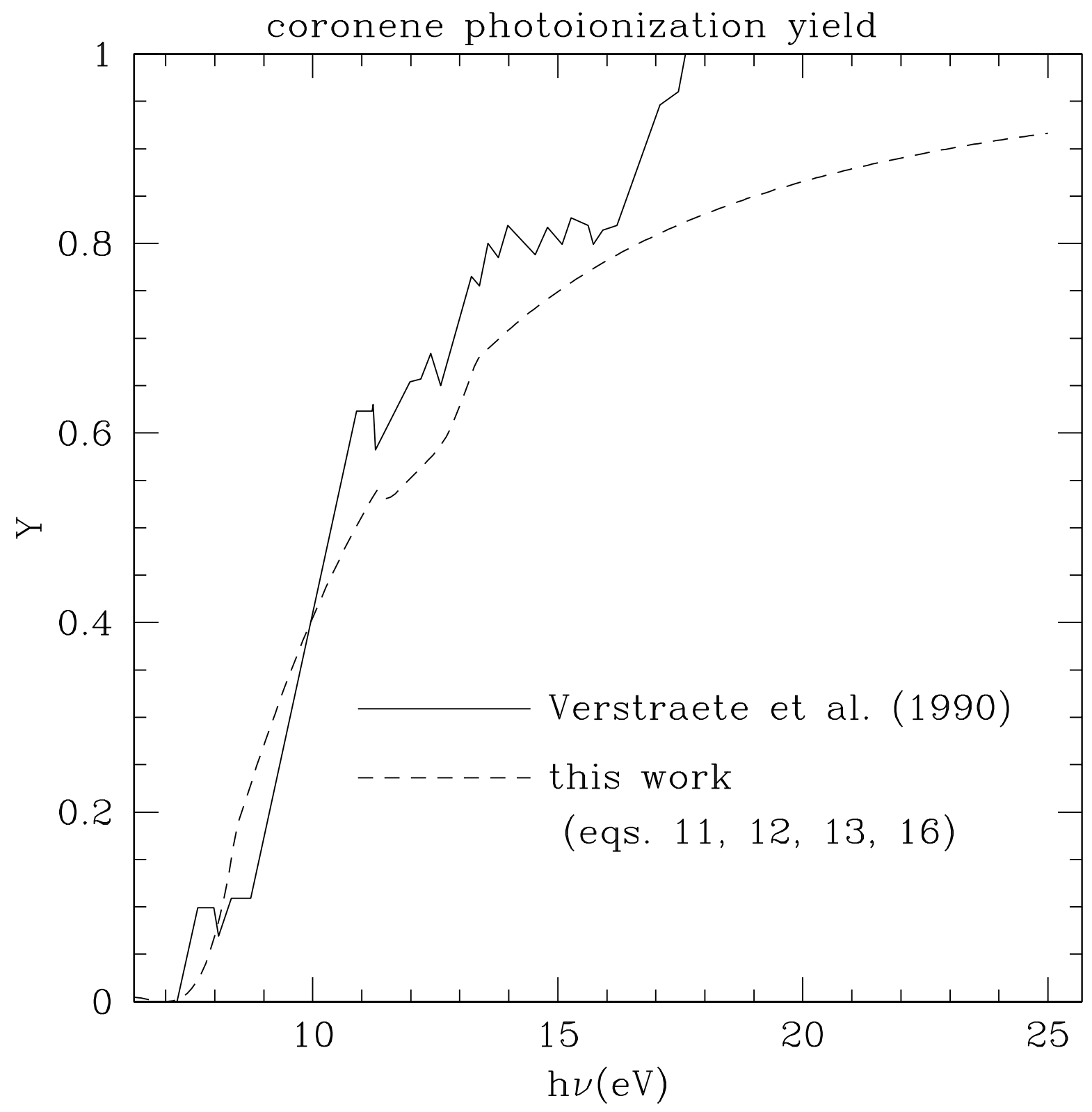

Fig. 4.- Photoionization yield for coronene, as measured by Verstraete et al. (1990) (solid) and as calculated using our model (dashed). 


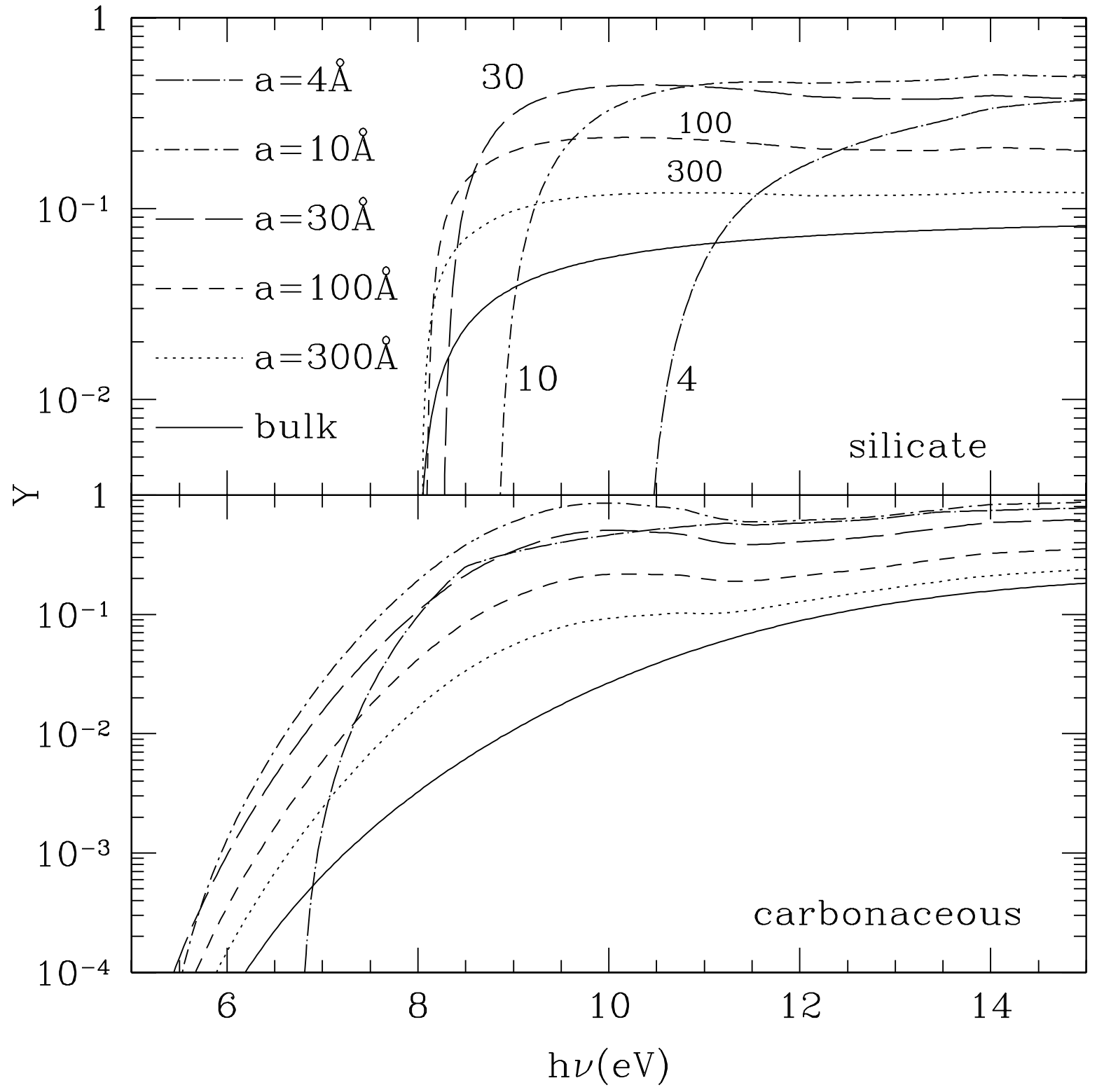

Fig. 5.- Photoelectric yield $Y$ for neutral graphite and silicate grains as a function of incident photon energy $h \nu$, for several values of the grain size $a$, as indicated. 


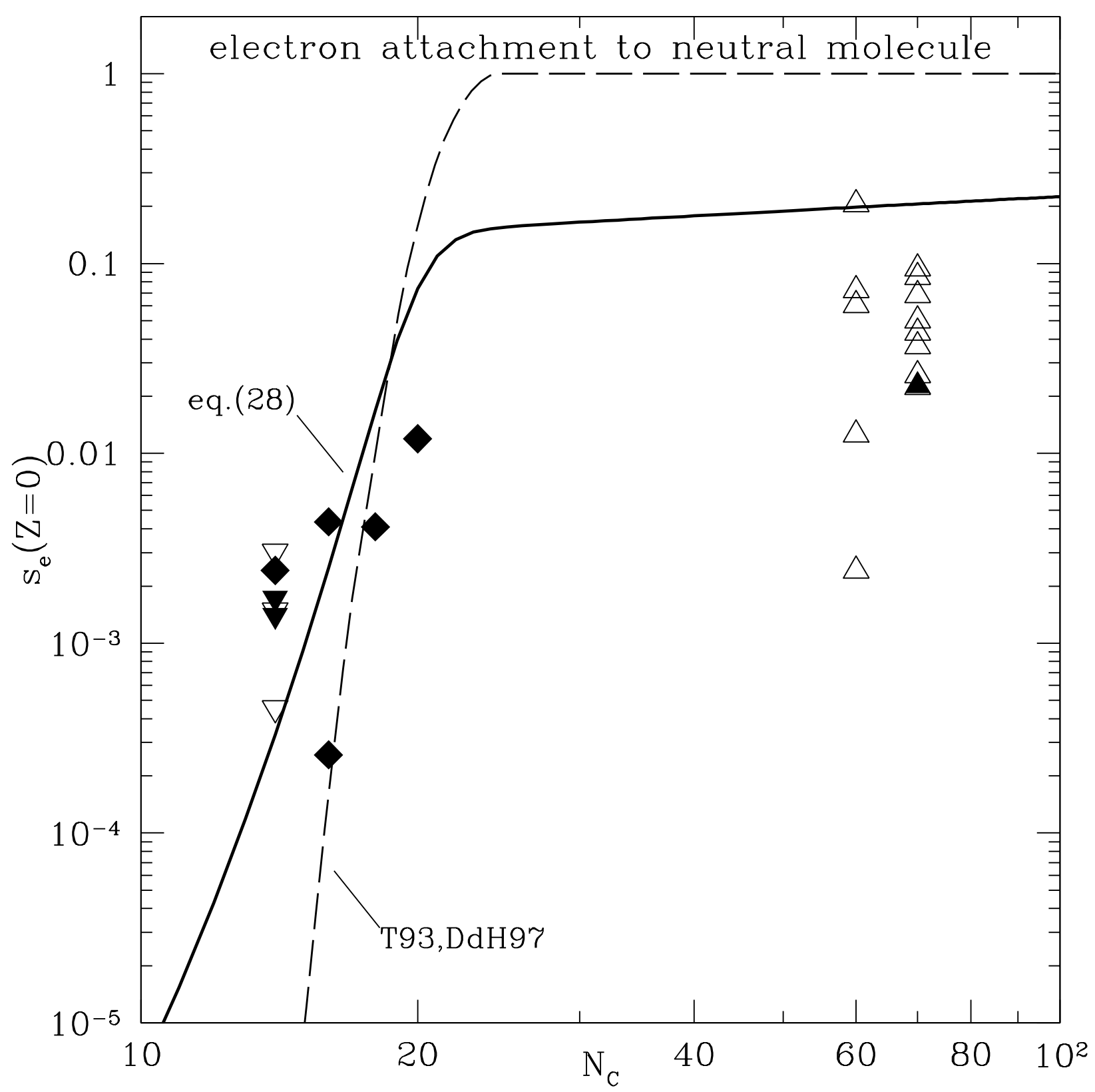

Fig. 6.- Sticking coefficients $s_{e}$ for electron attachment to neutral molecules, as a function of $N_{\mathrm{C}}$, the number of atoms other than $\mathrm{H}$ (mostly $\mathrm{C}$ ). Diamonds: acridine $\mathrm{C}_{13} \mathrm{H}_{9} \mathrm{~N}$, fluoranthene $\mathrm{C}_{16} \mathrm{H}_{10}$, pyrene $\mathrm{C}_{16} \mathrm{H}_{10}$, tetracene $\mathrm{C}_{18} \mathrm{H}_{12}$, and perylene $\mathrm{C}_{20} \mathrm{H}_{12}$ (Tobita et al. 1992). Triangles: anthracene $\mathrm{C}_{14} \mathrm{H}_{10}$ (Tobita et al. 1992; Canosa et al. 1994; Moustefaoui et al. 1998), $\mathrm{C}_{60}$ (Smith et al. 1993), and $\mathrm{C}_{70}$ (Spanel \& Smith 1994). Filled symbols are for $T=300 \mathrm{~K}$. Open symbols for $\mathrm{C}_{60}$ and $\mathrm{C}_{70}$ are for $T=500-4500 \mathrm{~K}$. Open symbols for anthracene are for $T=48-170 \mathrm{~K}$ (Moustefaoui et al. 1998). Solid curve: adopted empirical fit given by eq. (28). Broken curve: $s_{e}$ adopted by Tielens (1993) and Dartois \& d'Hendecourt (1997). 


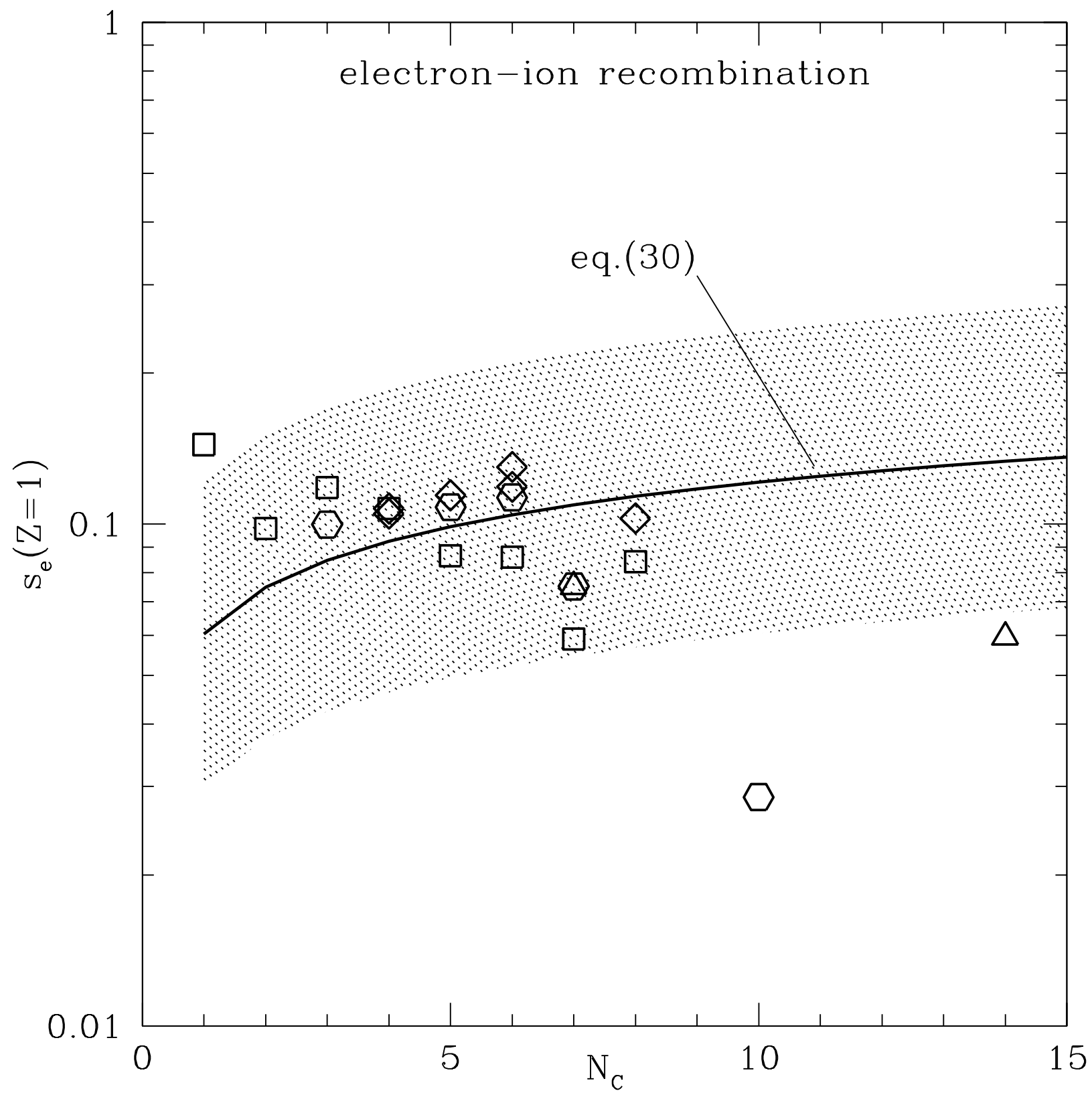

Fig. 7.- Sticking coefficients $s_{e}$ for electron recombination with positive ions. Hexagons: $T=$ $300 \mathrm{~K}$ experimental results of Abouelaziz et al. (1993) for $\mathrm{C}_{3} \mathrm{H}_{3}^{+}, \mathrm{C}_{5} \mathrm{H}_{3}^{+}, \mathrm{C}_{6} \mathrm{H}_{6}^{+}, \mathrm{C}_{7} \mathrm{H}_{5}^{+}$, and $\mathrm{C}_{10} \mathrm{H}_{8}^{+}$. Squares: $T=300 \mathrm{~K}$ results of Lehfaoui et al. (1997) for alkanes $\mathrm{CH}_{5}^{+}, \mathrm{C}_{2} \mathrm{H}_{5}^{+}, \mathrm{C}_{3} \mathrm{H}_{7}^{+}, \mathrm{C}_{4} \mathrm{H}_{9}^{+}, \mathrm{C}_{5} \mathrm{H}_{11}^{+}$, $\mathrm{C}_{6} \mathrm{H}_{13}^{+}, \mathrm{C}_{7} \mathrm{H}_{15}^{+}$, and $\mathrm{C}_{8} \mathrm{H}_{17}^{+}$. Triangle: $T=300 \mathrm{~K}$ results of Rowe et al. (1995) for $\mathrm{C}_{7} \mathrm{H}_{8}^{+}$and $\mathrm{C}_{14} \mathrm{H}_{10}^{+}$ phenanthrene. Diamonds: $T=300 \mathrm{~K}$ results of Rebrion-Rowe et al. (1998) for $\mathrm{C}_{4} \mathrm{H}_{5}^{+} \mathrm{C}_{4} \mathrm{H}_{11}^{+} \mathrm{C}_{5} \mathrm{H}_{9}^{+}$ $\mathrm{C}_{6} \mathrm{H}_{4}^{+} \quad \mathrm{C}_{6} \mathrm{H}_{5}^{+} \mathrm{C}_{8} \mathrm{H}_{7}^{+}$. Solid curve: adopted empirical fit given by eq. (30); shaded region is within factor of two. 


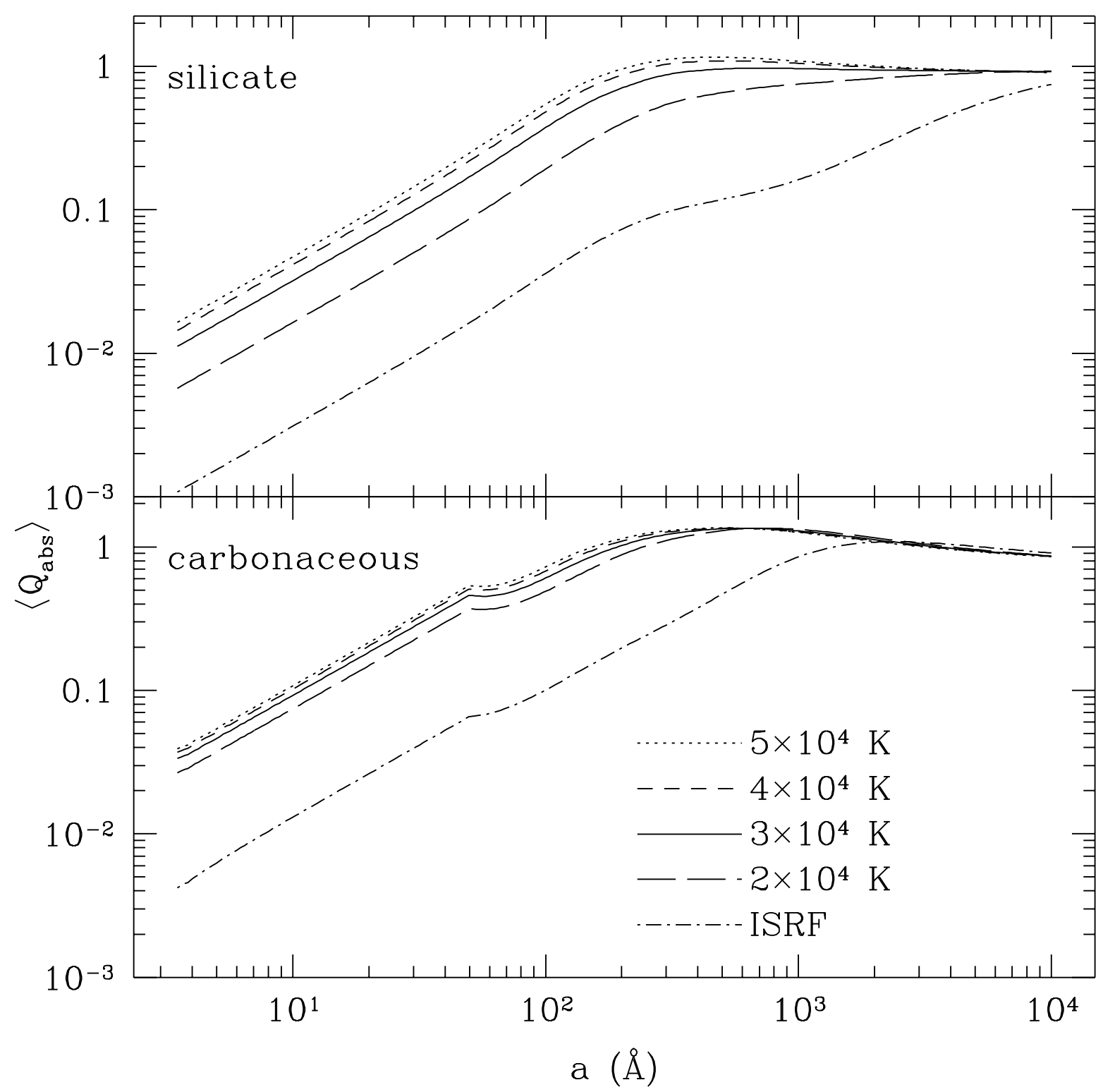

Fig. 8.- Absorption efficiency factors for neutral carbonaceous and silicate grains, averaged over the interstellar radiation field (ISRF) and blackbody spectra with indicated color temperatures (cut off at $13.6 \mathrm{eV}$ ). The kink at $a=50 \AA$ results from the Li \& Draine (2000) prescription for blending $\mathrm{PAH}$ and graphite optical properties. 


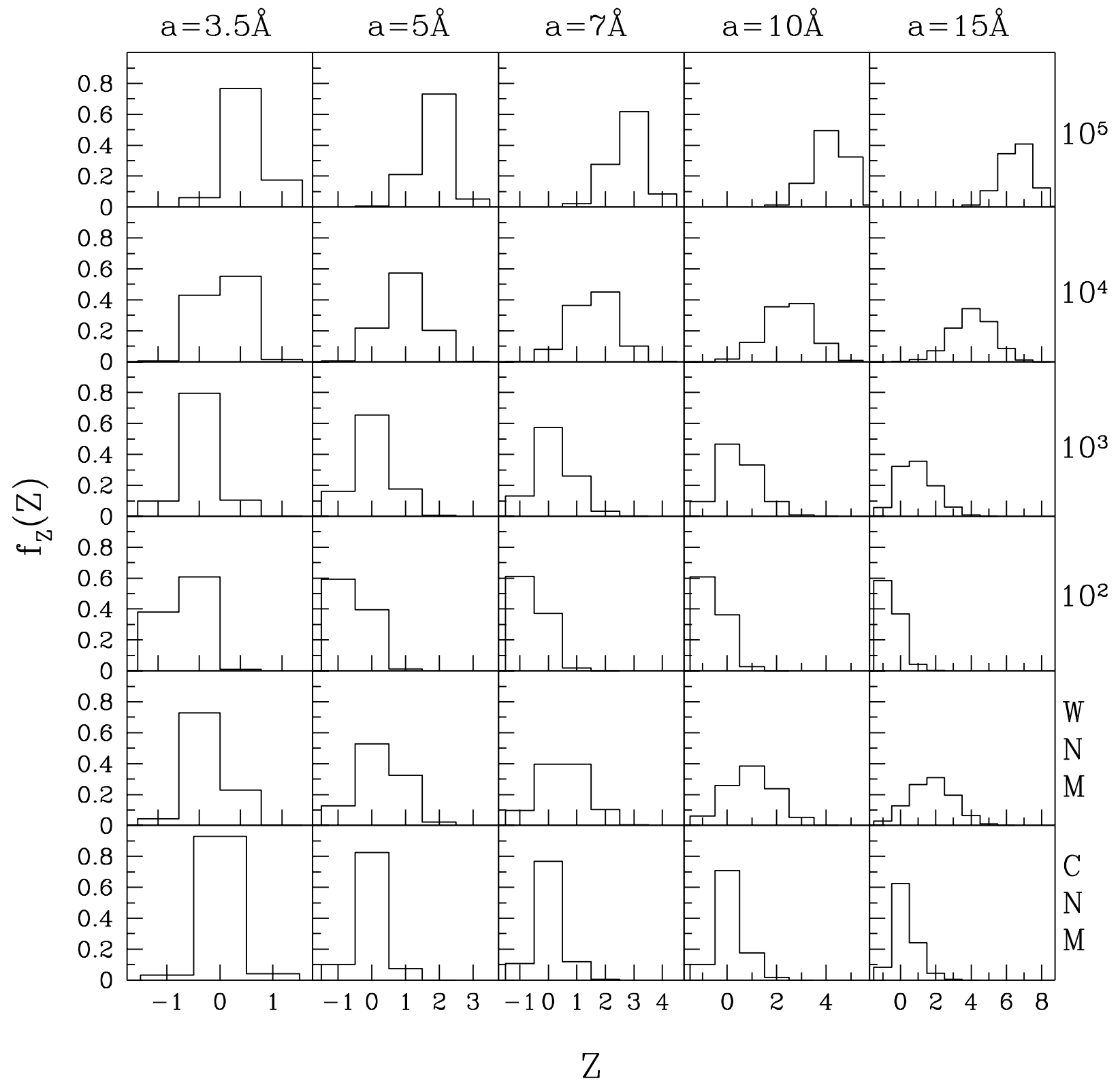

Fig. 9.- Charge distributions for carbonaceous grains with $a=3.5,5,7,10$, and $15 \AA$ and six sets of ambient conditions. For the upper four panels, we adopt a blackbody radiation spectrum (cut off at $13.6 \mathrm{eV}$ ) with $T_{\mathrm{c}}=3 \times 10^{4} \mathrm{~K}$ and $T=10^{3} \mathrm{~K}$; the values of $G \sqrt{T} / n_{e}$, in $\mathrm{K}^{1 / 2} \mathrm{~cm}^{3}$ are indicated. The bottom two panels are for the warm neutral medium (WNM) and cold neutral medium $(\mathrm{CNM})$. 


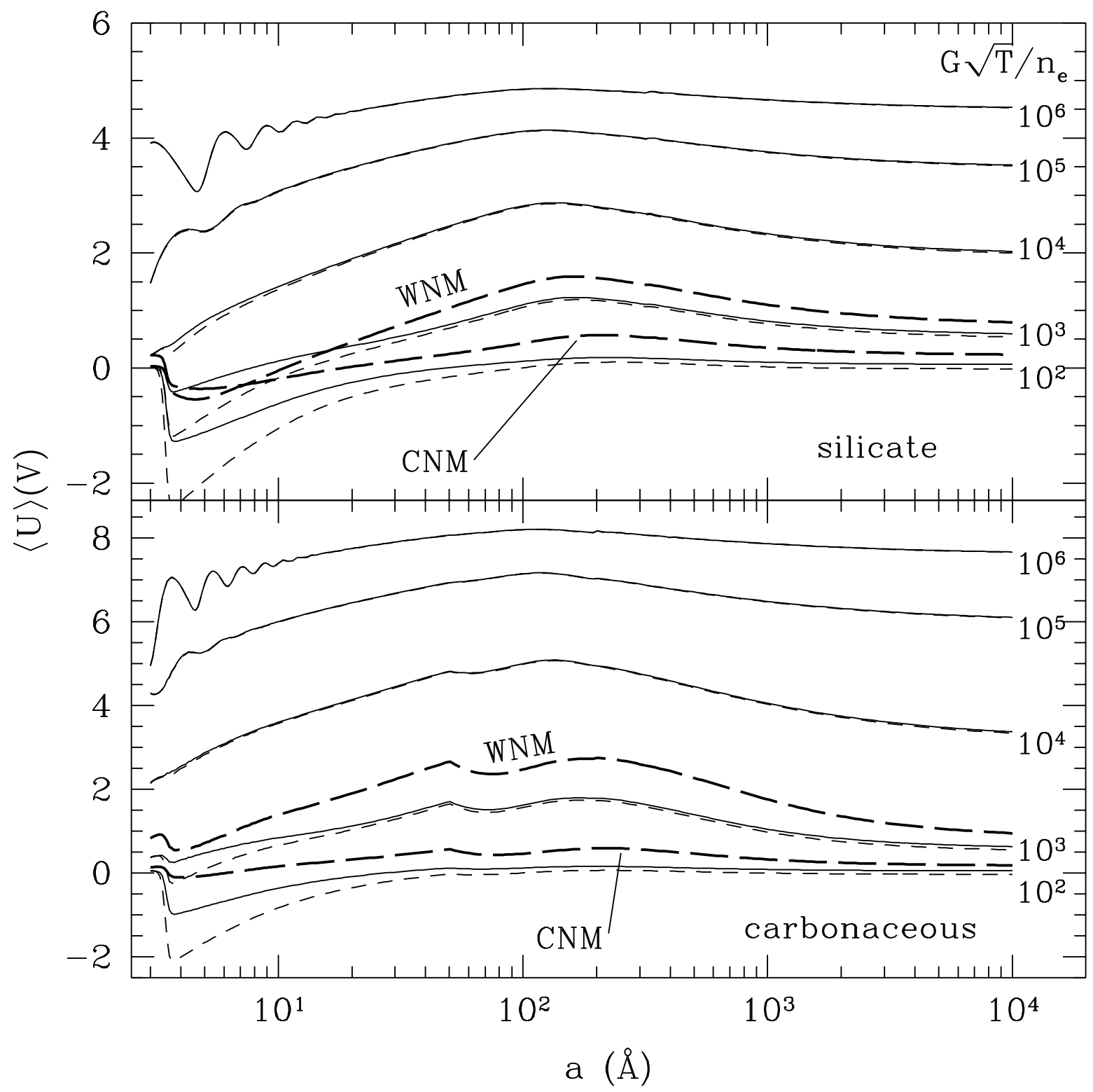

Fig. 10.- Average electrostatic potential $\langle U\rangle$ for a blackbody radiation field with $T_{\mathrm{c}}=3 \times 10^{4} \mathrm{~K}$ (cut off at $13.6 \mathrm{eV}$ ). The top (bottom) panel is for silicate (carbonaceous) grains; curves labelled CNM (WNM) are for cold (warm) neutral media; solid (short-dashed) lines indicate $T=100 \mathrm{~K}$ $(1000 \mathrm{~K})$. For the $T=100 \mathrm{~K}$ and $1000 \mathrm{~K}$ curves, values of $G \sqrt{T} / n_{e}$ are indicated in $\mathrm{K}^{1 / 2} \mathrm{~cm}^{3}$. 


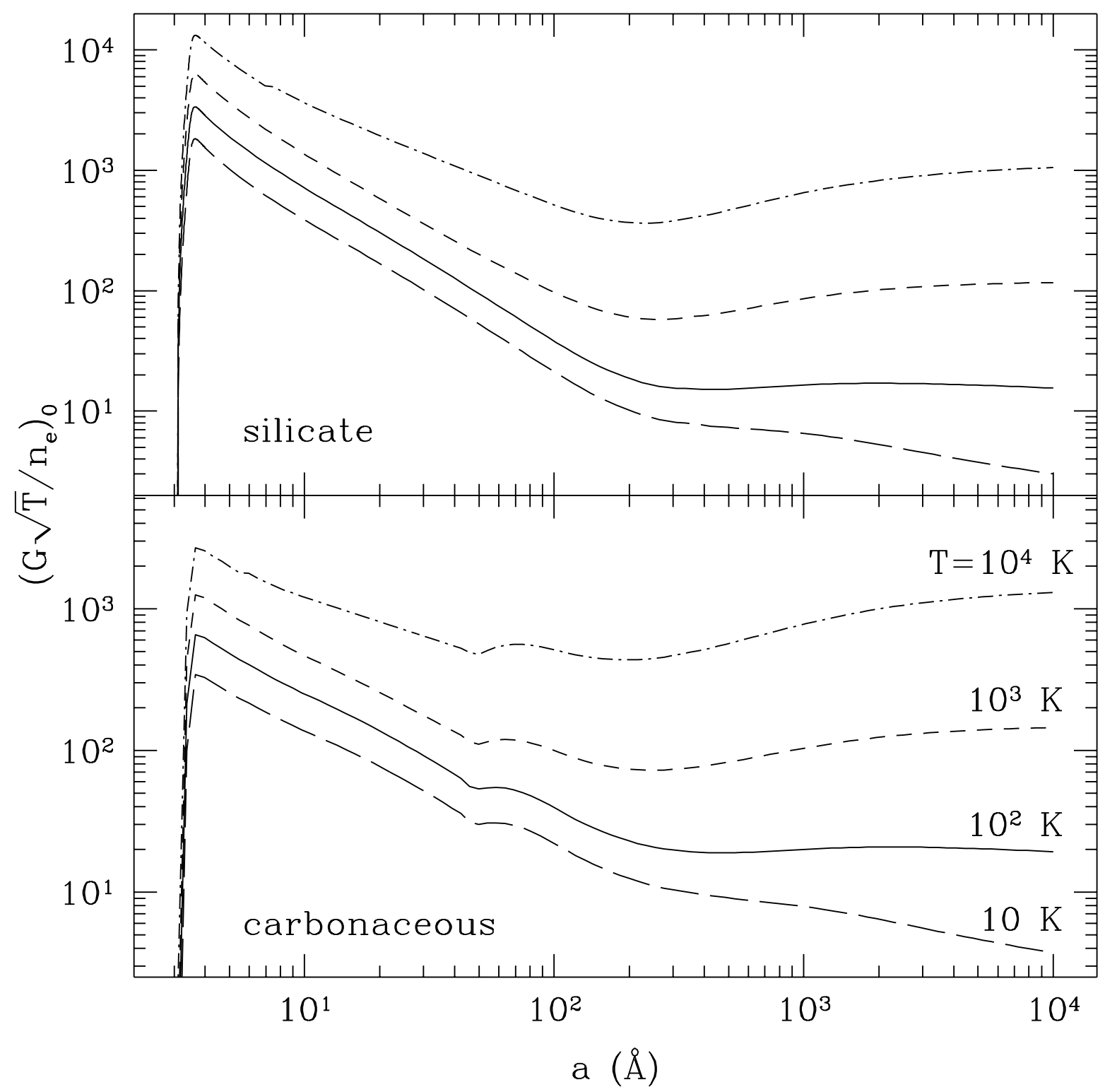

Fig. 11. - The value of the charging parameter for which $\langle Z\rangle=0$, for carbonaceous and silicate grains, a blackbody radiation field with $T_{\mathrm{c}}=3 \times 10^{4} \mathrm{~K}$ (cut off at $13.6 \mathrm{eV}$ ), and various gas temperatures, as labeled. 


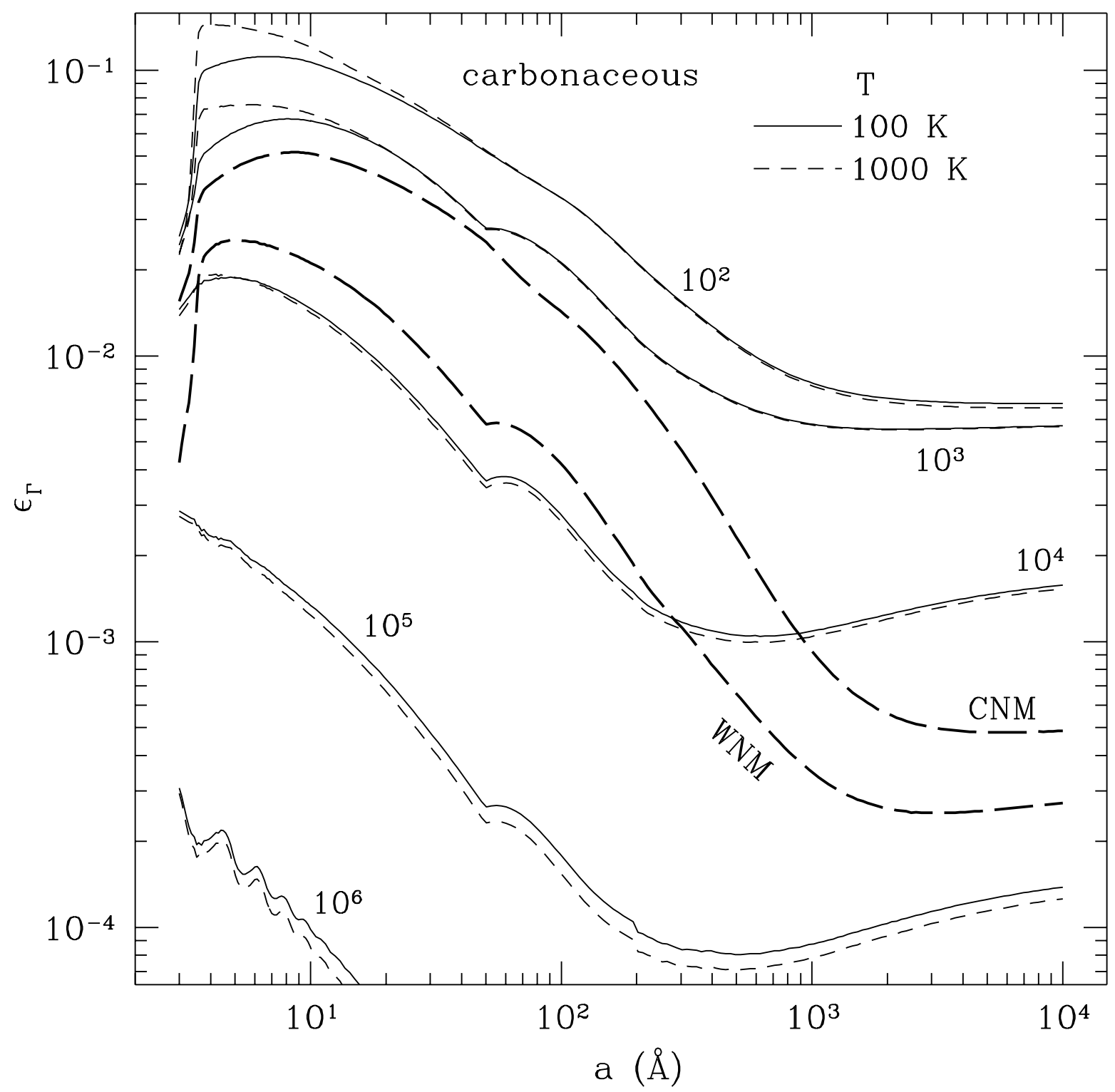

Fig. 12.- Gas heating efficiency for carbonaceous grains for two gas temperatures: $100 \mathrm{~K}$ (solid) and $10^{3} \mathrm{~K}$ (short-dashed), four values of $G \sqrt{T} / n_{e}$, as labelled, and $T_{\mathrm{c}}=3 \times 10^{4} \mathrm{~K}$. (The radiation is cut off at $13.6 \mathrm{eV}$.) Curves labelled CNM (WNM) are for cold (warm) neutral media and the ISRF. 


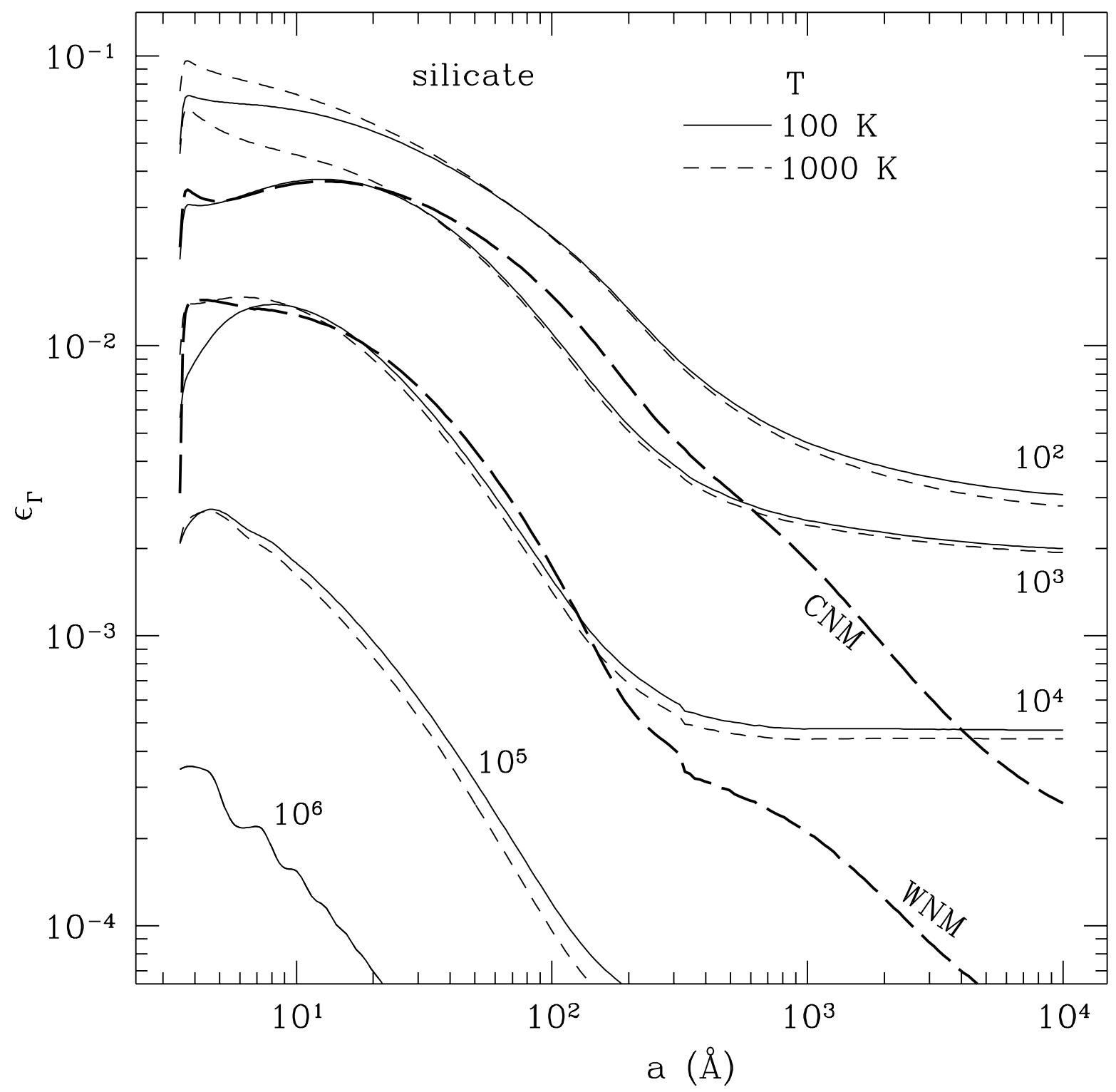

Fig. 13. - Same as Figure 12, but for silicate grains. The curve for $T=1000 \mathrm{~K}$ and $G \sqrt{T} / n_{e}=$ $10^{6} \mathrm{~K}^{1 / 2} \mathrm{~cm}^{3}$ lies entirely below the plot region. 


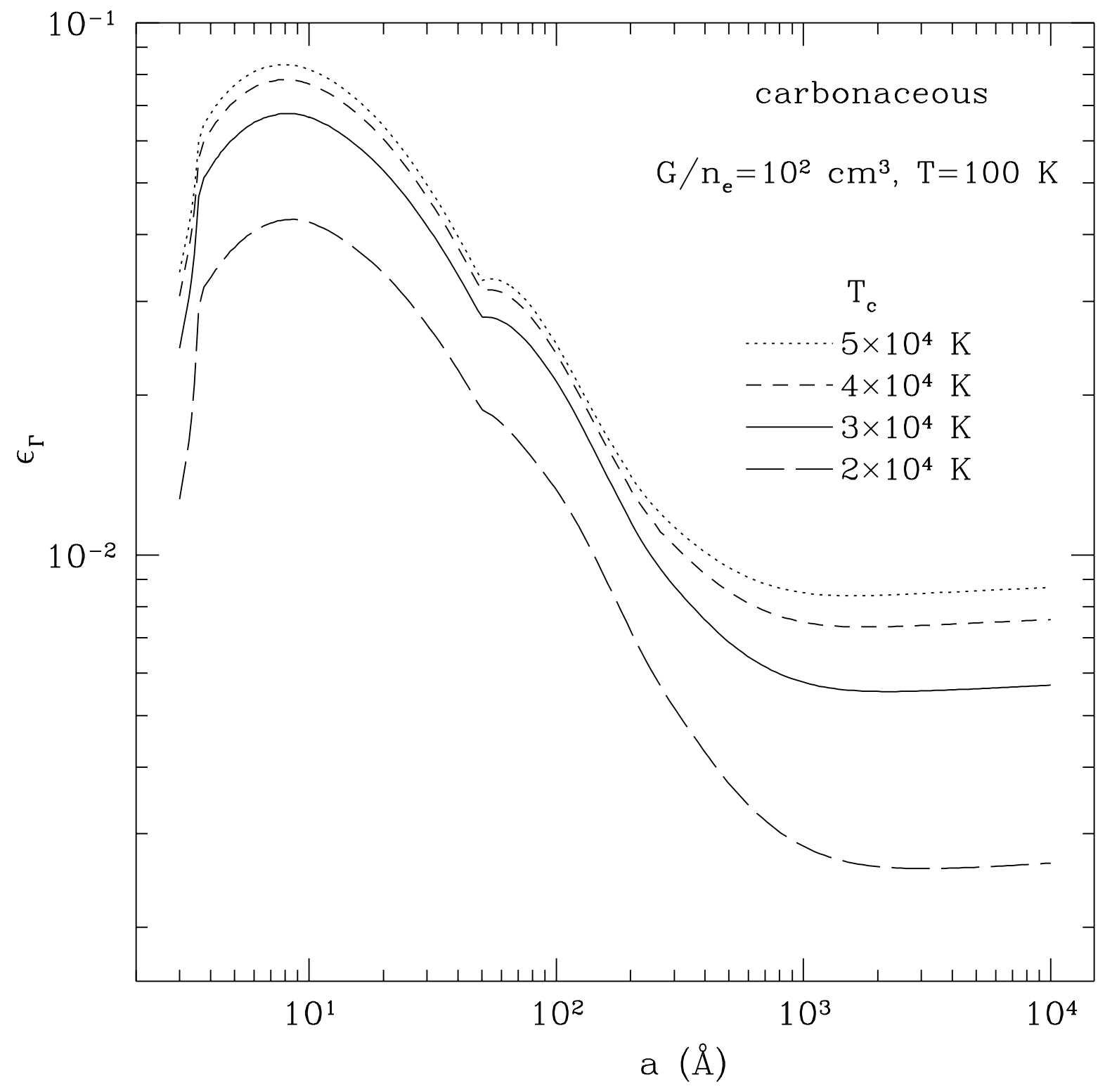

Fig. 14.- Gas heating efficiency for carbonaceous grains, $T=100 \mathrm{~K}, G \sqrt{T} / n_{e}=10^{3} \mathrm{~K}^{1 / 2} \mathrm{~cm}^{3}$, and four values of $T_{\mathrm{c}}$ as indicated. (The radiation is cut off at $13.6 \mathrm{eV}$.) 


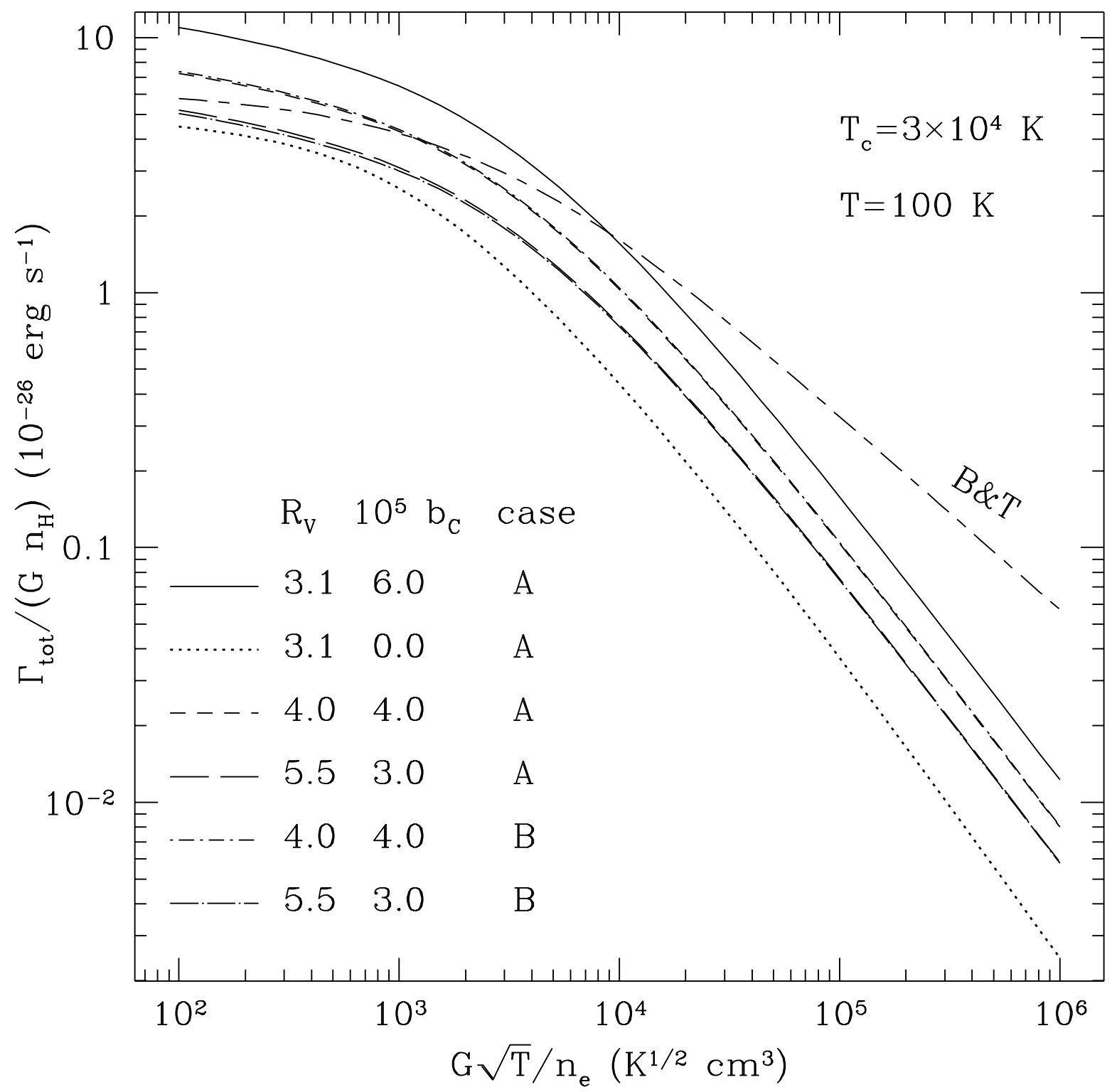

Fig. 15. - The photoelectric heating rate minus the cooling rate due to gas phase ions and electrons colliding with and sticking to the grains; for a blackbody radiation spectrum (cut off at $13.6 \mathrm{eV}$ ) with $T_{\mathrm{c}}=3 \times 10^{4} \mathrm{~K}$, gas temperature $T=100 \mathrm{~K}$, and various grain size distributions from Weingartner \& Draine (2000), as indicated. For comparison, we also display the Bakes \& Tielens (1994) result $(\mathrm{B} \& \mathrm{~T})$. 


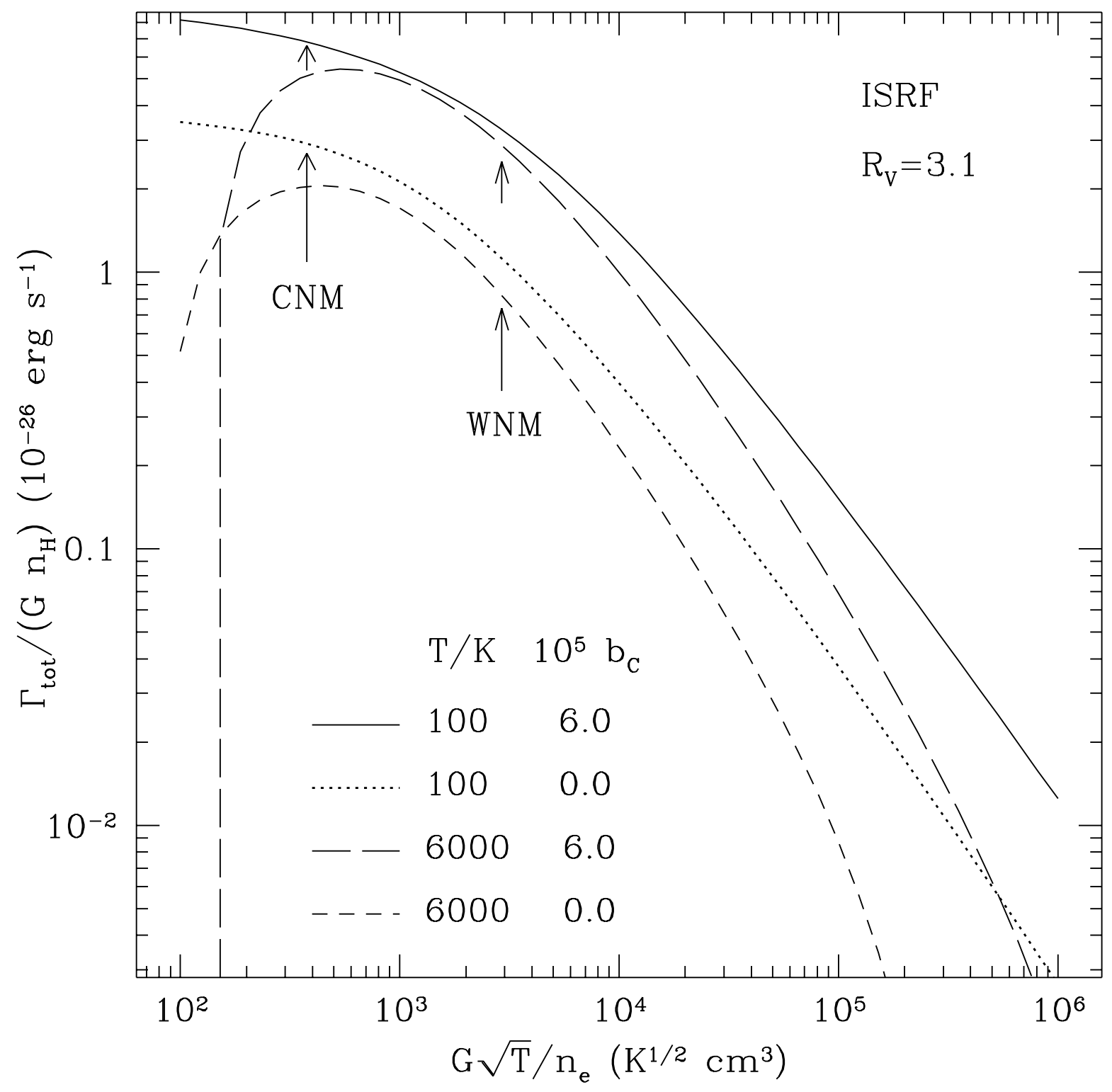

Fig. 16. - Same as Figure 15, but for the ISRF of equation (31), $R_{V}=3.1$, and various values of $b_{\mathrm{C}}$ and gas temperature $T$. 


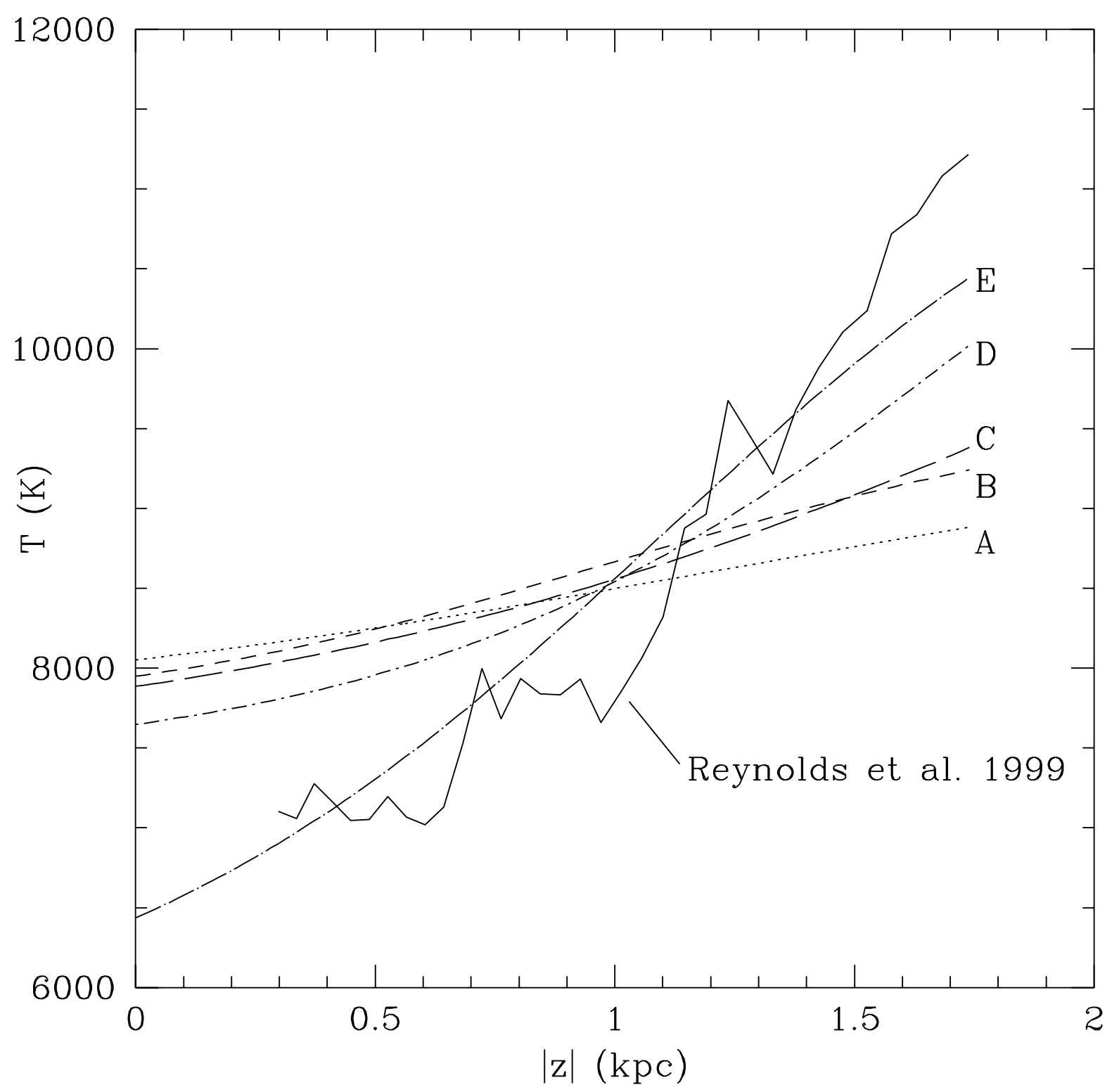

Fig. 17. - WIM temperature profiles as a function of distance from the Galactic midplane $|z|$. The solid curve was inferred by Reynolds et al. (1999) from observations of the $[\mathrm{N} \mathrm{II}] / \mathrm{H} \alpha$ line intensity ratio; the other curves are results of calculations including photoelectric heating, for various sets of parameter values as indicated in Table 6 . 


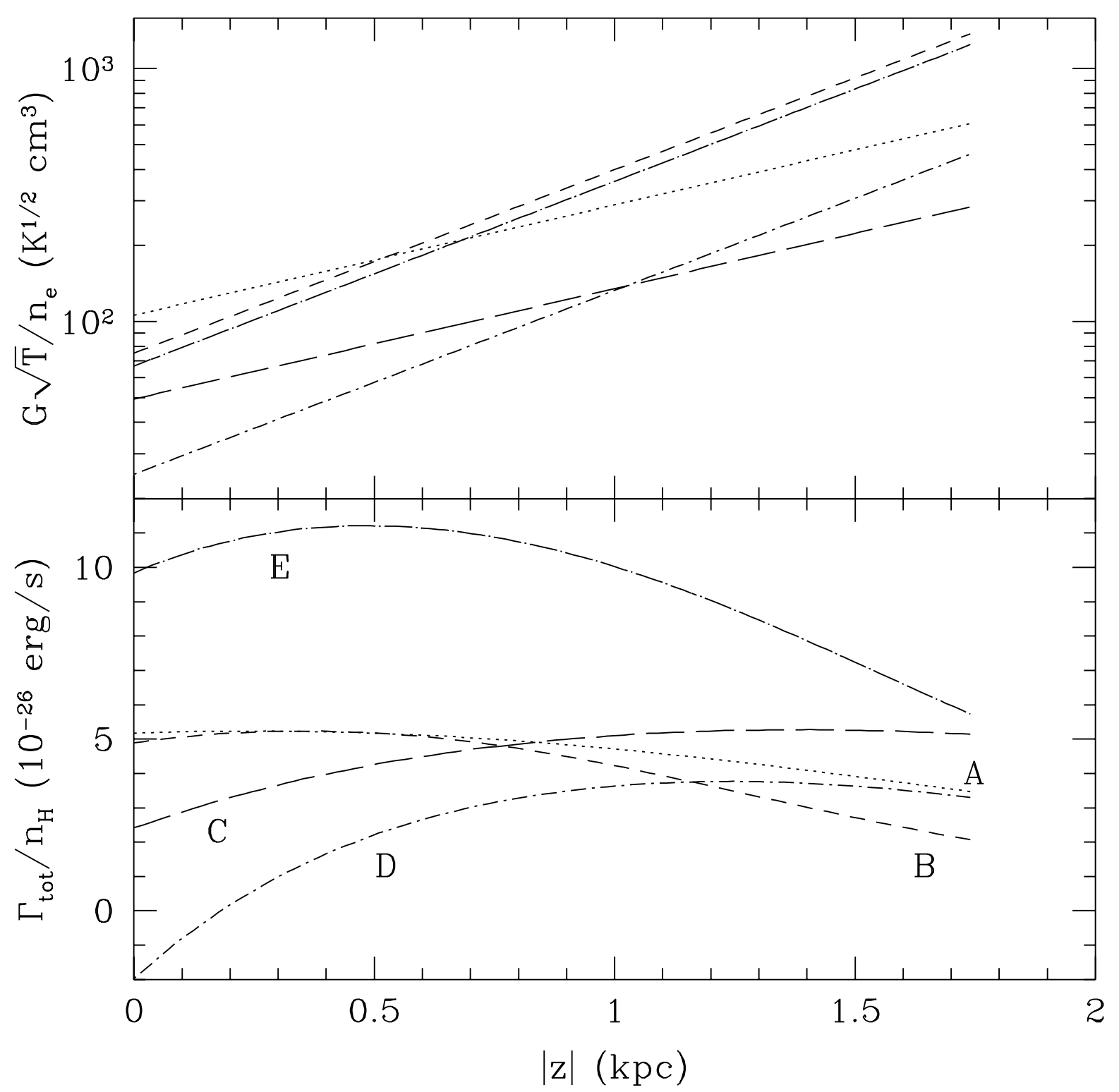

Fig. 18. - Upper panel: Values of the charging parameter as a function of $|z|$ for the five cases of Table 6. Lower panel: The ratio of the net grain heating rate (i.e. photoelectric heating minus collisional cooling) to the gas density as a function of $|z|$, for the five cases of Table 6 . 
Table 1. Blackbody Components of the ISRF (Mathis et al. 1983)

\begin{tabular}{ccc}
\hline \hline$i$ & $w_{i}$ & $T_{i} / \mathrm{K}$ \\
\hline 1 & $10^{-14}$ & 7500 \\
2 & $1.65 \times 10^{-13}$ & 4000 \\
3 & $4 \times 10^{-13}$ & 3000 \\
\hline
\end{tabular}


Table 2. Photoelectric Heating Parameters ${ }^{\mathrm{a}}$

\begin{tabular}{|c|c|c|c|c|c|c|c|c|c|c|c|c|}
\hline$R_{V}$ & $b_{\mathrm{C}}$ & case & rad field ${ }^{\mathrm{b}}$ & $C_{0}$ & $C_{1}$ & $C_{2}$ & $C_{3}$ & $C_{4}$ & $C_{5}$ & $C_{6}$ & err & $h_{s}{ }^{\mathrm{c}}$ \\
\hline 3.1 & 0.0 & $\mathrm{~A}$ & B0 & 5.56 & $1.82 \times 10^{-2}$ & 0.00492 & 0.03368 & 0.557 & 0.666 & 0.532 & 0.15 & 0.75 \\
\hline 3.1 & 2.0 & $\mathrm{~A}$ & B0 & 3.41 & 4.27 & 0.03700 & 0.00569 & 0.102 & 0.494 & 0.669 & 0.19 & 0.89 \\
\hline 3.1 & 4.0 & $\mathrm{~A}$ & B0 & 10.59 & $4.05 \times 10^{-3}$ & 0.01234 & 0.01391 & 0.808 & 0.580 & 0.573 & 0.19 & 0.94 \\
\hline 3.1 & 6.0 & $\mathrm{~A}$ & B0 & 3.66 & 7.66 & 0.00661 & 0.01552 & 0.094 & 0.697 & 0.482 & 0.20 & 0.96 \\
\hline 4.0 & 0.0 & $\mathrm{~A}$ & B0 & 4.30 & $1.79 \times 10^{-4}$ & 0.00572 & 0.02488 & 1.026 & 0.701 & 0.505 & 0.15 & 0.77 \\
\hline 4.0 & 2.0 & $\mathrm{~A}$ & B0 & 5.03 & 2.27 & 0.07441 & 0.00345 & 0.140 & 0.412 & 0.737 & 0.18 & 0.92 \\
\hline 4.0 & 4.0 & $\mathrm{~A}$ & B0 & 3.28 & 4.44 & 0.00786 & 0.01219 & 0.102 & 0.686 & 0.500 & 0.20 & 0.96 \\
\hline 5.5 & 0.0 & $\mathrm{~A}$ & B0 & 3.67 & $2.42 \times 10^{-3}$ & 0.07034 & 0.00360 & 0.725 & 0.365 & 0.814 & 0.15 & 0.77 \\
\hline 5.5 & 1.0 & $\mathrm{~A}$ & B0 & 5.45 & $2.44 \times 10^{-2}$ & 0.09594 & 0.00225 & 0.534 & 0.418 & 0.758 & 0.18 & 0.89 \\
\hline 5.5 & 2.0 & $\mathrm{~A}$ & B0 & 4.87 & $7.28 \times 10^{-3}$ & 0.00812 & 0.01969 & 0.656 & 0.620 & 0.534 & 0.19 & 0.94 \\
\hline 5.5 & 3.0 & $\mathrm{~A}$ & B0 & 5.93 & $9.26 \times 10^{-2}$ & 0.00675 & 0.01648 & 0.417 & 0.683 & 0.489 & 0.20 & 0.96 \\
\hline 4.0 & 0.0 & B & B0 & 4.85 & $6.59 \times 10^{-2}$ & 0.00437 & 0.04234 & 0.415 & 0.664 & 0.508 & 0.16 & 0.86 \\
\hline 4.0 & 2.0 & B & B0 & 5.97 & 0.287 & 0.00736 & 0.02065 & 0.302 & 0.637 & 0.528 & 0.18 & 0.93 \\
\hline 4.0 & 4.0 & B & B0 & 7.97 & 0.175 & 0.00277 & 0.03820 & 0.382 & 0.771 & 0.401 & 0.19 & 0.96 \\
\hline 5.5 & 0.0 & B & B0 & 4.04 & 0.653 & 0.11310 & 0.00259 & 0.198 & 0.348 & 0.809 & 0.18 & 0.89 \\
\hline 5.5 & 1.0 & B & B0 & 3.38 & 0.608 & 0.00396 & 0.02568 & 0.192 & 0.752 & 0.439 & 0.18 & 0.93 \\
\hline 5.5 & 2.0 & B & B0 & 1.10 & 3.30 & 0.00941 & 0.01431 & 0.087 & 0.643 & 0.524 & 0.20 & 0.95 \\
\hline 5.5 & 3.0 & B & B0 & 3.47 & 1.77 & 0.00494 & 0.01953 & 0.140 & 0.723 & 0.456 & 0.20 & 0.97 \\
\hline 3.1 & 0.0 & $\mathrm{~A}$ & ISRF & 4.35 & $2.63 \times 10^{-5}$ & 0.00242 & 0.03003 & 1.235 & 0.827 & 0.399 & 0.15 & 0.73 \\
\hline 3.1 & 2.0 & $\mathrm{~A}$ & ISRF & 4.87 & 0.948 & 0.02576 & 0.00766 & 0.188 & 0.490 & 0.650 & 0.18 & 0.88 \\
\hline 3.1 & 4.0 & $\mathrm{~A}$ & ISRF & 7.41 & 0.772 & 0.03895 & 0.00606 & 0.239 & 0.408 & 0.708 & 0.19 & 0.94 \\
\hline 3.1 & 6.0 & $\mathrm{~A}$ & ISRF & 9.30 & 0.248 & 0.00697 & 0.01848 & 0.365 & 0.633 & 0.509 & 0.20 & 0.96 \\
\hline
\end{tabular}

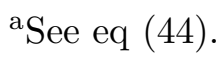

${ }^{\mathrm{b}} \mathrm{B} 0$ refers to a blackbody spectrum with $T_{\mathrm{c}}=3 \times 10^{4} \mathrm{~K}$ and the ISRF of Mathis et al. (1983) is defined in eq (31).

${ }^{\mathrm{c}}$ Fraction of the total heating due to grains with $a<100 \AA$, for $G \sqrt{T} / n_{e}=10^{2} \mathrm{~K}^{1 / 2} \mathrm{~cm}^{3}$ and $T=100 \mathrm{~K}$. 
Table 3. Collisional Cooling Parameters ${ }^{\mathrm{a}}$

\begin{tabular}{llllllllll}
\hline \hline$R_{V}$ & $b_{\mathrm{C}}$ & case & rad field & $D_{0}$ & $D_{1}$ & $D_{2}$ & $D_{3}$ & $D_{4}$ & err \\
\hline 3.1 & 0.0 & A & B0 & 0.4800 & 1.783 & -7.617 & 1.655 & 0.06326 & 0.17 \\
3.1 & 2.0 & A & B0 & 0.5322 & 1.325 & -6.047 & 1.435 & 0.05378 & 0.14 \\
3.1 & 4.0 & A & B0 & 0.4336 & 2.108 & -7.359 & 1.736 & 0.06502 & 0.14 \\
3.1 & 6.0 & A & B0 & 0.4270 & 2.120 & -7.301 & 1.786 & 0.06731 & 0.15 \\
4.0 & 0.0 & A & B0 & 0.5332 & 1.379 & -7.125 & 1.504 & 0.05732 & 0.17 \\
4.0 & 2.0 & A & B0 & 0.4425 & 2.019 & -7.755 & 1.712 & 0.06424 & 0.14 \\
4.0 & 4.0 & A & B0 & 0.4847 & 1.493 & -6.109 & 1.528 & 0.05751 & 0.13 \\
5.5 & 0.0 & A & B0 & 0.4184 & 2.129 & -9.082 & 1.818 & 0.06943 & 0.16 \\
5.5 & 1.0 & A & B0 & 0.4981 & 1.510 & -7.237 & 1.535 & 0.05777 & 0.14 \\
5.5 & 2.0 & A & B0 & 0.4851 & 1.501 & -6.775 & 1.533 & 0.05762 & 0.13 \\
5.5 & 3.0 & A & B0 & 0.4948 & 1.442 & -6.377 & 1.505 & 0.05645 & 0.13 \\
4.0 & 0.0 & B & B0 & 0.4568 & 1.876 & -7.810 & 1.688 & 0.06412 & 0.15 \\
4.0 & 2.0 & B & B0 & 0.4597 & 1.594 & -6.675 & 1.599 & 0.06094 & 0.14 \\
4.0 & 4.0 & B & B0 & 0.5155 & 1.340 & -5.791 & 1.449 & 0.05411 & 0.13 \\
5.5 & 0.0 & B & B0 & 0.4360 & 1.996 & -8.372 & 1.735 & 0.06572 & 0.15 \\
5.5 & 1.0 & B & B0 & 0.5140 & 1.349 & -6.676 & 1.472 & 0.05536 & 0.14 \\
5.5 & 2.0 & B & B0 & 0.4633 & 1.690 & -7.158 & 1.607 & 0.06055 & 0.14 \\
5.5 & 3.0 & B & B0 & 0.4677 & 1.780 & -7.173 & 1.615 & 0.06027 & 0.13 \\
3.1 & 0.0 & A & ISRF & 0.4291 & 2.406 & -8.357 & 1.714 & 0.06354 & 0.15 \\
3.1 & 2.0 & A & ISRF & 0.5232 & 1.678 & -5.942 & 1.339 & 0.04813 & 0.14 \\
3.1 & 4.0 & A & ISRF & 0.3959 & 2.380 & -6.554 & 1.575 & 0.05674 & 0.13 \\
3.1 & 6.0 & A & ISRF & 0.3632 & 2.937 & -7.601 & 1.742 & 0.06228 & 0.12 \\
\hline
\end{tabular}

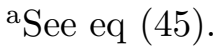

${ }^{\mathrm{b}} \mathrm{B} 0$ refers to a blackbody spectrum with $T_{\mathrm{c}}=3 \times 10^{4} \mathrm{~K}$ and the ISRF of Mathis et al. (1983) is defined in eq (31). 
Table 4. Photoelectric Heating Rates for H II Regions

\begin{tabular}{|c|c|c|c|c|c|c|c|c|c|c|}
\hline \multirow[b]{2}{*}{$R_{V}^{\mathrm{d}}$} & \multirow[b]{2}{*}{$10^{5} b_{\mathrm{C}}^{\mathrm{e}}$} & \multirow[b]{2}{*}{ case } & \multirow[b]{2}{*}{$T_{\mathrm{c}}^{\mathrm{f}}$} & \multirow[b]{2}{*}{$G / n_{\mathrm{H}}=$} & \multicolumn{3}{|c|}{$\Gamma_{\mathrm{pe}} / G n_{\mathrm{H}}{ }^{\mathrm{a}, \mathrm{b}}$} & \multicolumn{3}{|c|}{$\Gamma_{\mathrm{pe}} / G n_{\mathrm{H}}{ }^{\mathrm{a}, \mathrm{c}}$} \\
\hline & & & & & $0.1^{\mathrm{g}}$ & 1.0 & 10. & 0.1 & 1.0 & 10. \\
\hline 3.1 & 0.0 & $\mathrm{~A}$ & 3.5 & & 1.03 & 0.83 & 0.40 & 3.58 & 2.91 & 1.25 \\
\hline 3.1 & 6.0 & $\mathrm{~A}$ & 3.5 & & 2.35 & 2.06 & 1.16 & 8.15 & 7.07 & 3.59 \\
\hline 4.0 & 4.0 & $\mathrm{~A}$ & 3.5 & & 1.54 & 1.36 & 0.76 & 5.37 & 4.66 & 2.35 \\
\hline 5.5 & 3.0 & $\mathrm{~A}$ & 3.5 & & 1.09 & 0.97 & 0.55 & 3.83 & 3.34 & 1.68 \\
\hline 4.0 & 4.0 & B & 3.5 & & 1.57 & 1.38 & 0.77 & 5.49 & 4.76 & 2.38 \\
\hline 5.5 & 3.0 & B & 3.5 & & 1.06 & 0.94 & 0.54 & 3.74 & 3.28 & 1.64 \\
\hline 3.1 & 0.0 & $\mathrm{~A}$ & 4.5 & & 1.13 & 0.91 & 0.43 & 5.93 & 4.73 & 1.80 \\
\hline 3.1 & 6.0 & $\mathrm{~A}$ & 4.5 & & 2.57 & 2.26 & 1.24 & 13.1 & 11.1 & 5.02 \\
\hline 4.0 & 4.0 & $\mathrm{~A}$ & 4.5 & & 1.68 & 1.48 & 0.82 & 8.62 & 7.31 & 3.25 \\
\hline 5.5 & 3.0 & $\mathrm{~A}$ & 4.5 & & 1.19 & 1.06 & 0.59 & 6.13 & 5.21 & 2.31 \\
\hline 4.0 & 4.0 & B & 4.5 & & 1.72 & 1.51 & 0.83 & 8.81 & 7.46 & 3.30 \\
\hline 5.5 & 3.0 & B & 4.5 & & 1.16 & 1.03 & 0.58 & 5.96 & 5.08 & 2.26 \\
\hline
\end{tabular}

${ }^{\mathrm{a}} 10^{-25} \mathrm{erg} \mathrm{s}^{-1}$

${ }^{\mathrm{b}}$ Blackbody spectrum cut off at $13.6 \mathrm{eV}$

${ }^{\mathrm{c}}$ Full blackbody spectrum

${ }^{\mathrm{d}} R_{V}=A_{V} / E_{B-V}$, ratio of visual extinction to reddening

${ }^{\mathrm{e}} \mathrm{C}$ abundance in very small grain population

${ }^{\mathrm{f}} 10^{4} \mathrm{~K}$

${ }^{\text {g} V a l u e ~ f o r ~} G / n_{\mathrm{H}}$, in $\mathrm{cm}^{3}$ 
Table 5. Recombination Cooling Rates for H II Regions

\begin{tabular}{|c|c|c|c|c|c|c|c|c|c|}
\hline \multirow[b]{2}{*}{$R_{V}^{\mathrm{d}}$} & \multirow[b]{2}{*}{$b_{\mathrm{C}}{ }^{\mathrm{e}}$} & \multirow[b]{2}{*}{ case } & \multirow[b]{2}{*}{$G / n_{\mathrm{H}}=$} & \multicolumn{3}{|c|}{$\Lambda / G n_{\mathrm{H}}{ }^{\mathrm{a}, \mathrm{b}}$} & \multicolumn{3}{|c|}{$\Lambda / G n_{\mathrm{H}}{ }^{\mathrm{a}, \mathrm{c}}$} \\
\hline & & & & $0.1^{\mathrm{g}}$ & 1.0 & 10. & 0.1 & 1.0 & 10. \\
\hline 3.1 & 0.0 & $\mathrm{~A}$ & 3.5 & 7.43 & 1.02 & 0.22 & 7.91 & 1.36 & 0.36 \\
\hline 3.1 & 4.0 & $\mathrm{~A}$ & 3.5 & 29.4 & 3.47 & 0.64 & 30.2 & 4.17 & 1.00 \\
\hline 4.0 & 2.0 & $\mathrm{~A}$ & 3.5 & 18.7 & 2.20 & 0.41 & 19.2 & 2.66 & 0.64 \\
\hline 5.5 & 1.0 & A & 3.5 & 13.2 & 1.55 & 0.29 & 13.6 & 1.87 & 0.45 \\
\hline 4.0 & 2.0 & B & 3.5 & 18.9 & 2.23 & 0.42 & 19.4 & 2.69 & 0.65 \\
\hline 5.5 & 1.0 & B & 3.5 & 13.1 & 1.53 & 0.28 & 13.5 & 1.84 & 0.44 \\
\hline 3.1 & 0.0 & $\mathrm{~A}$ & 4.5 & 7.46 & 1.05 & 0.23 & 8.29 & 1.58 & 0.44 \\
\hline 3.1 & 4.0 & $\mathrm{~A}$ & 4.5 & 29.4 & 3.51 & 0.67 & 30.9 & 4.68 & 1.21 \\
\hline 4.0 & 2.0 & $\mathrm{~A}$ & 4.5 & 18.7 & 2.23 & 0.43 & 19.6 & 2.98 & 0.78 \\
\hline 5.5 & 1.0 & $\mathrm{~A}$ & 4.5 & 13.2 & 1.57 & 0.30 & 13.9 & 2.10 & 0.55 \\
\hline 4.0 & 2.0 & B & 4.5 & 18.9 & 2.26 & 0.43 & 19.9 & 3.02 & 0.79 \\
\hline 5.5 & 1.0 & B & 4.5 & 13.1 & 1.55 & 0.29 & 13.8 & 2.06 & 0.54 \\
\hline
\end{tabular}

${ }^{\mathrm{a}} 10^{-25} \mathrm{erg} \mathrm{s}^{-1}$

${ }^{\mathrm{b}}$ Blackbody spectrum cut off at $13.6 \mathrm{eV}$

${ }^{\mathrm{c}}$ Full blackbody spectrum

${ }^{\mathrm{d}} R_{V}=A_{V} / E_{B-V}$, ratio of visual extinction to reddening

${ }^{\mathrm{e}} \mathrm{C}$ abundance in very small grain population

${ }^{\mathrm{f}} 10^{4} \mathrm{~K}$

${ }^{\text {g}}$ Value for $G / n_{\mathrm{H}}$, in $\mathrm{cm}^{3}$ 
Table 6. WIM Heating Examples

\begin{tabular}{ccccc}
\hline \hline Case & Grain Size Distribution & WIM Filling Factor & $\begin{array}{c}\Gamma_{\mathrm{pi}} / n_{e}^{2} T_{4}^{-0.8} \\
10^{-24} \mathrm{erg} \mathrm{cm} \mathrm{s}^{-1}\end{array}$ & $G$ \\
\hline $\mathrm{A}$ & $R_{V}=3.1, b_{\mathrm{C}}=0.0$ & 0.2 & 1.5 & 0.3 \\
$\mathrm{~B}$ & $R_{V}=3.1, b_{\mathrm{C}}=0.0$ & $0.1 \exp |z| / 750 \mathrm{pc}$ & 1.5 & 0.3 \\
$\mathrm{C}$ & $R_{V}=3.1, b_{\mathrm{C}}=6 \times 10^{-5}$ & 0.2 & 1.5 & 0.14 \\
$\mathrm{D}$ & $R_{V}=3.1, b_{\mathrm{C}}=6 \times 10^{-5}$ & $0.1 \exp |z| / 750 \mathrm{pc}$ & 1.5 & 0.1 \\
$\mathrm{E}$ & $R_{V}=3.1, b_{\mathrm{C}}=6 \times 10^{-5}$ & $0.1 \exp |z| / 750 \mathrm{pc}$ & 0.7 & 0.27 \\
\hline
\end{tabular}

